ARTICLE

https://doi.org/10.1038/s41467-019-10925-3

\title{
Adult stem cell deficits drive S/c29a3 disorders in mice
}

Sreenath Nair ${ }^{1}$, Anne M. Strohecker ${ }^{2,3}$, Avinash K. Persaud1, Bhawana Bissa1, Shanmugam Muruganandan', Craig McElroy', Rakesh Pathak1, Michelle Williams ${ }^{4}$, Radhika Raj ${ }^{1}$, Amal Kaddoumi ${ }^{5}$, Alex Sparreboom', Aaron M. Beedle ${ }^{6} \&$ Rajgopal Govindarajan ${ }^{1,7}$

Mutations exclusively in equilibrative nucleoside transporter 3 (ENT3), the only intracellular nucleoside transporter within the solute carrier 29 (SLC29) gene family, cause an expanding spectrum of human genetic disorders (e.g., $\mathrm{H}$ syndrome, PHID syndrome, and SHML/RDD syndrome). Here, we identify adult stem cell deficits that drive ENT3-related abnormalities in mice. ENT3 deficiency alters hematopoietic and mesenchymal stem cell fates; the former leads to stem cell exhaustion, and the latter leads to breaches of mesodermal tissue integrity. The molecular pathogenesis stems from the loss of lysosomal adenosine transport, which impedes autophagy-regulated stem cell differentiation programs via misregulation of the AMPK-mTOR-ULK axis. Furthermore, mass spectrometry-based metabolomics and bioenergetics studies identify defects in fatty acid utilization, and alterations in mitochondrial bioenergetics can additionally propel stem cell deficits. Genetic, pharmacologic and stem cell interventions ameliorate ENT3-disease pathologies and extend the lifespan of ENT3-deficient mice. These findings delineate a primary pathogenic basis for the development of ENT3 spectrum disorders and offer critical mechanistic insights into treating human ENT3related disorders.

\footnotetext{
${ }^{1}$ Division of Pharmaceutics and Pharmaceutical Chemistry, College of Pharmacy, Ohio State University, Columbus, OH 43210, USA. ${ }^{2}$ Department of Cancer Biology and Genetics, College of Medicine, Ohio State University, Columbus, OH 43210, USA. ${ }^{3}$ Molecular Biology and Cancer Genetics, Ohio State University Comprehensive Cancer Center, Ohio State University, Columbus, OH 43210, USA. ${ }^{4}$ Department of Radiology, Ohio State University, Columbus, OH 43210, USA. ${ }^{5}$ Department of Drug Discovery and Development, Harrison School of Pharmacy, Auburn University, Auburn, AL 36849, USA. ${ }^{6}$ Department of Pharmaceutical Sciences, SUNY Binghamton University, Binghamton, NY 13902, USA. ${ }^{7}$ Translational Therapeutics, Ohio State University Comprehensive Cancer Center, Ohio State University, Columbus, OH 43210, USA. Correspondence and requests for materials should be addressed to R.G. (email: govindarajan.21@osu.edu)
} 
series of human genetic disorders with dermatological, musculoskeletal, endocrinological, and hematological abnormalities have been reported over the past decades under various diagnostic terms, namely gonodermatosis, pigmented hypertrichosis with insulin-dependent diabetes mellitus (PHID), familial histiocytosis (FHC), sinus histiocytosis with massive lymphadenopathy (SHML), Rosai-Dorfman Disease (RDD), and familial plasmacytosis ${ }^{1-4}$. Despite the clinical similarities, the genotype-to-phenotype link and molecular pathogenesis remain unknown. In 2008, three single-nucleotide changes in SLC29A3 (1279 G > A, $1309 \mathrm{G}>\mathrm{A}, 1045 \mathrm{delC})$ were reported as causative for an autosomal-recessive gonodermatosis called ' $\mathrm{H}$ syndrome' 5 . $\mathrm{H}$ syndrome refers to hyperpigmentation, hypertrichosis, hepatosplenomegaly, heart anomalies, hearing loss, hypogonadism, low-height, and hyperglycemia ${ }^{5}$. In 2009 , five mutations in SLC29A3 (347 T $>$ G, 940delT, $1309 \mathrm{G}>\mathrm{A}$, $1330 \mathrm{G}>\mathrm{T}$, and $1346 \mathrm{C}>\mathrm{G}$ ) were documented as causative for $\mathrm{PHID}^{6}$. PHID is associated with diabetes mellitus, lipodystrophy, and pigmented hypertrichosis with abnormal hematopoietic and myelofibrotic features ${ }^{6}$. In 2010, three mutations in SLC29A3 $(300+1 \mathrm{G}>\mathrm{A}, 307 \mathrm{delT}, 1309 \mathrm{G}>\mathrm{A})$ were reported for FHC, SHML/FHC, and RDD syndromes, which exhibit stunted growth, lymphadenopathy, and histiocytosis ${ }^{7}$. Presently, more than 20 disease-causing mutations in SLC29A3 and a broader spectrum of clinical manifestations have been reported ${ }^{8-16}$.

The SLC29 gene family encodes four equilibrative nucleoside transporters (ENTs; ENT1 to ENT4) that facilitate the membrane translocation of hydrophilic nucleosides to regulate salvage DNA synthesis and purinergic signaling ${ }^{17}$. The SLC29A3 gene (encoding ENT3) on chromosome 10 contains six exons, with mutation sites concentrated in the last exon encoding almost the entire carboxyl-half of ENT3 ${ }^{18}$. ENT3 is unique as the only intracellular nucleoside transporter in the family with putative localization in late endosomes, lysosomes, and mitochondria (other human ENTs (hENTs) primarily function at the cell surface $)^{19,20}$. In 2012, the roles of ENT3 in lysosomal homeostasis and macrophage biology were elucidated ${ }^{21}$. While impaired macrophage dysfunction accounts for some pathology in ENT3 disorders (e.g., histiocytosis), the molecular mechanisms of most disease manifestations are unknown. Nonetheless, the monogenic nature of ENT3 disorders suggests additional unifying mechanisms underlying ENT3 disease pathologies. Previous studies in our laboratory demonstrated that SLC29A3 disease mutations impair nucleoside transport, subcellular localization, protein stability, and $\mathrm{pH}$ sensing ability $18,20,22,23$. Studies from other laboratories also indicated a correlation between human disease severity and residual hENT3 activity because mutations with a partial loss of function have hypomorphic features ${ }^{24}$. Recently, the transport features and developmental expression pattern of the mouse ortholog of hENT3 (mENT3) were characterized ${ }^{7,19}$. Intriguingly, mENT3 showed similar kinetic features to hENT3 and tissue expression consistent with the pattern of dysfunction in humans.

Here, we establish a functional link between ENT3 and the AMPK-mTOR-ULK-regulated lysosome-autophagy pathway and demonstrate that adult stem cell deficits primarily drive Slc29a3 disorders in mice because of the loss of lysosomal transport of adenosine, a cargo transported through ENT3. Genetic, pharmacological, and stem cell interventions ameliorate the disease severity in mice, with therapeutic implications for human disorders.

\section{Results}

Slc29a3 ${ }^{-I-}$ mice recapitulate human phenotypes. The mENT3 knockout by gene trap of exons 1 and 2 (Slc29a3-/-; B6N.129S5-
Slc29a3tm1Lex/Mmcd) generated mice with an apparently normal phenotype until 10-12 weeks of age ${ }^{21}$. However, mice exhibited profound deterioration of health after 12 weeks with overt manifestations of several seemingly unrelated disorders resulting in $\sim 90 \%$ mortality at $18-20$ weeks. At 12 weeks of age, Slc29a3 $3^{-1-}$ mice demonstrated retarded growth and endurance capacity (Fig. 1a-c). Approximately $50-75 \%$ of mice displayed a characteristic 'hunchback" kyphosis, hypertrichosis, malocclusion, and skeletal deformities consisting of impaired bone and cartilage development (Fig. 1a, d, e and Supplementary Fig. 1a, b). The microCT analysis identified a loss of trabecular bone mineral density (Fig. 1f, g). A third of Slc29a3-1- mice presented with penile or vaginal prolapse with the mineralization of soft tissues (Fig. 1h). EchoMRI and necropsy revealed reductions in fat and lean mass (Fig. 1i, j), and elevated plasma lactate dehydrogenase suggested the presence of muscle damage (Fig. 1k). Many Slc29a3 ${ }^{-1}$ mice demonstrated hypogonadism (Supplementary Fig. 1c) and infertility, and, if bred, had 2-3 pups/litter with quick cessation of breeding. Mass spectrometry analysis of mouse plasma revealed endocrinopathy as a possible source of reproductive deficiency (Fig. 11).

At necropsy, the bone medulla was pale in appearance with reduced bone marrow cellularity (Fig. $1 \mathrm{~m}, \mathrm{n}$ ) reminiscent of abnormal hematopoietic features reported in humans ${ }^{25}$. The spleen (and occasionally liver) showed massive enlargements (Supplementary Fig. 1d) ${ }^{21}$. Histopathological examination revealed severe histiocytosis in numerous organs ${ }^{21}$ (Supplementary Fig. 1e). Additionally, the spleen, lymph nodes, and thymus showed lymphoid cell death (Supplementary Fig. 1f). Enlarged lymph nodes also showed architectural effacement with sinusoidal infiltration of histiocytes admixed with tingible body macrophages (Supplementary Fig. 1f). Blood parameters revealed anemia, hemoglobinemia, thrombocytopenia, and leukocytosis (Fig. 10). Overall, the abnormalities in Slc29a3 ${ }^{-1-}$ mice displayed striking similarities to several clinical features reported in human SLC29A3 disorders (Supplementary Fig. 2).

Stem cell deficits drive ENT3-related dysfunctions. Adult stem cells are crucial for the maintenance and repair of adult tissues ${ }^{26}$. The phenotypes observed in several mesenchymal (bone, cartilage, muscle, fat) and hematopoietic (blood cells and bloodforming tissues) tissues (Fig. 1 and Supplementary Fig. 1) suggested a possible defect arising from common adult mesenchymal stem cells (MSCs) and hematopoietic stem cells (HSCs). Therefore, we investigated the putative roles for ENT3 in the differentiation capabilities of MSCs and HSCs. When forced to differentiate into each of the mesenchymal lineages, Slc29a3 ${ }^{-1-}$ MSCs exhibited distinct deficits in forming mesenchymal lineages, osteoblasts, adipocytes, myocytes, and chondrocytes (Fig. 2a), with the reduction in lineage-specific markers (Fig. 2b). These findings suggested a common defect in ENT3-deficient MSCs for lineage specification resulting in widespread defects. Likewise, when Slc29a3 ${ }^{-/}$HSCs were induced to differentiate into multiple lineages, a distinct skewing toward myeloid (G/M/GM/ GEMM) over erythroid (E) commitment was observed (Fig. 2c). The induction of CFU-GEMM indicated that HSC differentiation is altered from hierarchically more primitive cell types ${ }^{27}$; however, the reciprocal induction of G/M/GM factors at the expense of E factors (Fig. 2d) also suggested a role for transcriptional reprogramming in skewing the balance toward myeloid production $^{28}$. Nevertheless, the identification of bona fide erythroid-tomyeloid skewing provided a compelling explanation for the exacerbated formation of histiocytes and leukocytes with accompanied anemia and thrombocytopenia in $S l c 29 a 3^{-1-}$ mice. Consistent with the increased ability of HSCs to differentiate into 
a

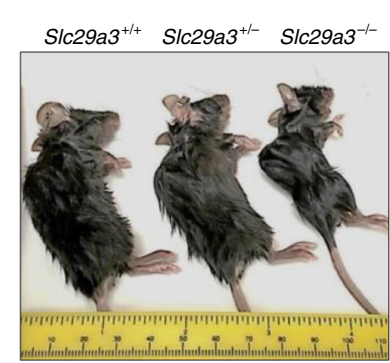

b

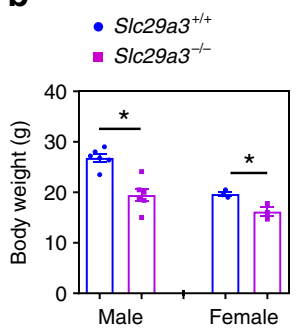

C

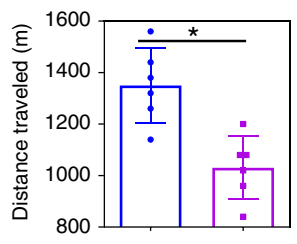

d

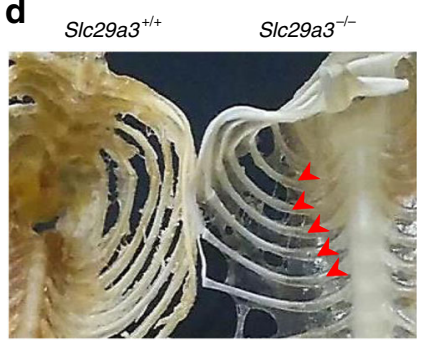

h

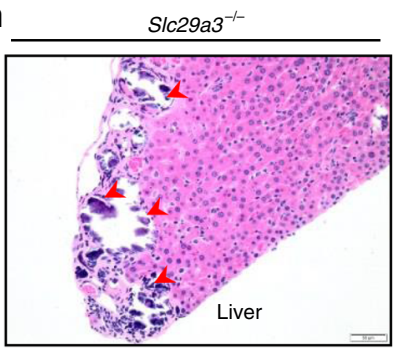

e

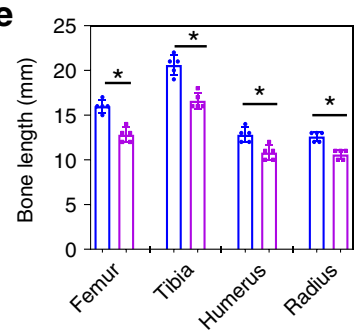

f
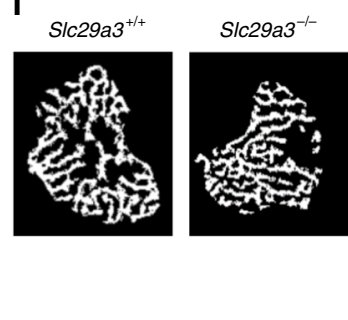

g
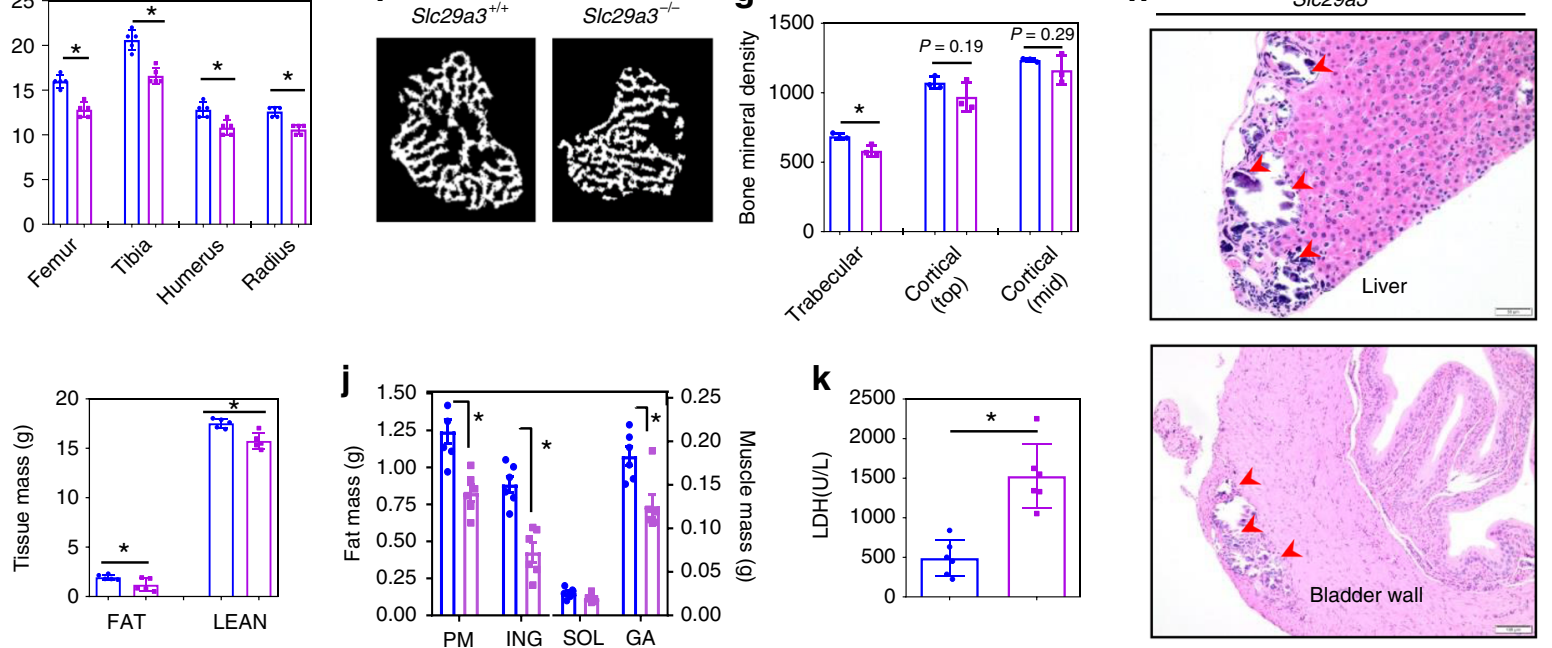

I
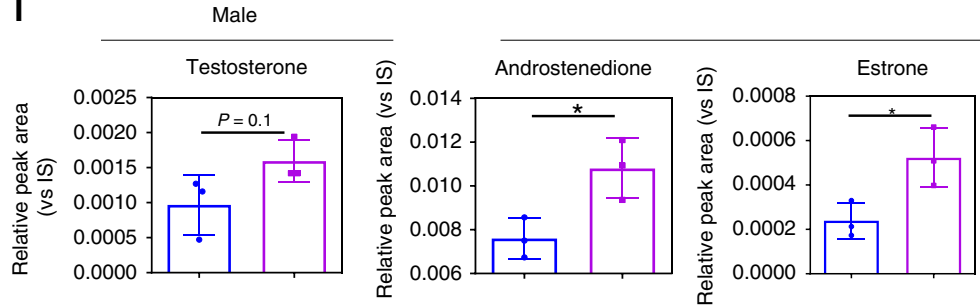

Female

m

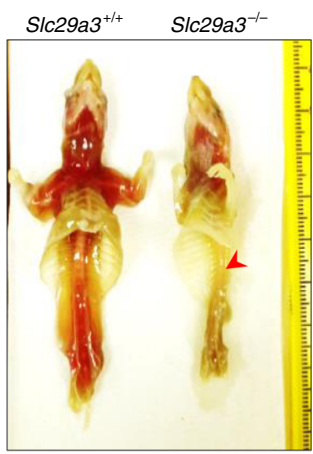

n

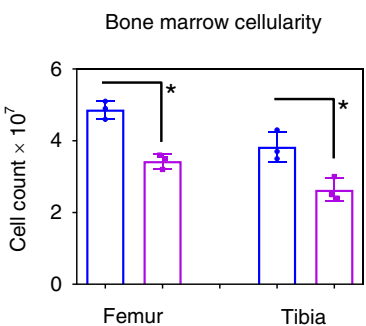

o

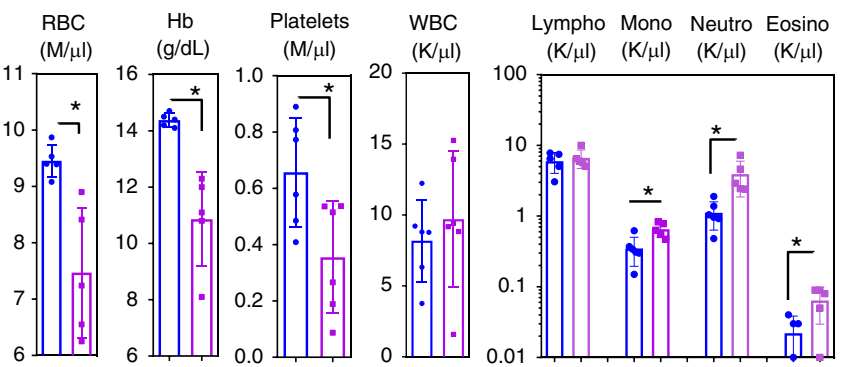

CFU-M, increased osteoclastogenesis from CFU-M (Fig. 2e, f) further explained the occurrence of skeletal deformities, aberrant bone mineralization, and ectopic mineralization in Slc29a3 $3^{-1-}$ mice.

To understand the nature of ENT3-related dysfunctions, we evaluated the behavior of Slc29a3 $3^{-1-}$ MSCs and HSCs in culture. Primary Slc29a3 ${ }^{-1-}$ MSCs exhibited rapid exhaustion of clonogenic potential within $2-3$ passages (Fig. $3 a$ ) with a near-complete loss of MSC-specific marker profiles (Fig. 3b and Supplementary Fig. 3a $)^{29}$. By contrast, Slc29a3 $3^{-1-}$ HSCs exhibited an enhanced proliferative capacity (Fig. 3c). However, the progenies lacking
HSC markers (Fig. 3d and Supplementary Fig. 3b) also indicated the loss of HSC clonogenicity. Consistently, Slc29a3-1- MSCs and HSCs showed decreased expression of genes (Fig. 3e) essential to maintain multipotentiality ${ }^{29,30}$. In vivo analysis of bone marrow revealed an apparent loss of stem cells beginning at $\sim 12$ weeks that reached a classical state of stem cell exhaustion at $\sim 16$ weeks (Fig. 3f and Supplementary Fig. 4a). To test whether the lack of ENT3 is a mechanism underlying the exhaustion of bone marrow stem cell populations, we examined the ability of WT and Slc29a3 $3^{-1-}$ HSCs for hematopoietic reconstitution in a uniform C57BL6/J-congenic 129S5/SvEvBrd mouse line. We 
Fig. $1 \mathrm{~S} / \mathrm{c} 29 a 3^{-/-}$mice recapitulate the hENT3 disease features. S/c29a3-/- mice presented a weak, hunched-back appearance (a) and weighed less than Slc29a3 ${ }^{+/+}$mice (males, $n=6$; females, $n=3$; mean \pm SEM) (b). Treadmill run time to exhaustion $(n=6$, mean \pm SEM) (c). Translucent appearance of ribs in S/c29a3-/- mice (d). Lengths of the femur, tibia, humerus, and radius measured using a Vernier caliper $(n=5$, mean \pm SEM) (e). Bone microarchitecture and mineral density assessed by microCT analysis (f) show reduced trabecular bone mineral density in S/c29a3-/- mice $(n=3$, mean \pm SEM) (g). Displays ectopic mineralization of the liver and bladder wall (purple; liver, Scale bar: $50 \mu \mathrm{m}$; bladder wall, Scale bar: $100 \mu \mathrm{m})$ in S/c9a3-/- mice (h). EchoMRI analysis of body fat and lean tissue composition $(n=5$, mean \pm SEM) (i). Weights of freshly isolated parametrial (PM) and inguinal (ING) fat pads (left axis), and soleus (SOL) and gastrocnemius (GA) skeletal muscles (right axis) ( $n=6$, mean \pm SEM) (j). Increased plasma LDH levels in SIc29a3 ${ }^{-/}$mice $(n=6$, mean \pm SEM) (k). Mass spectrometric analysis of sex steroid hormones (males, $n=3$; females, $n=3$, mean \pm SEM). IS, internal standard (I). Medullary hematopoiesis examined at necropsy $(\mathbf{m})$ and bone marrow cellularity $(\mathbf{n})(n=3$, mean $\pm \operatorname{SEM})$. RBC counts $(n=5$, mean \pm SEM), hemoglobin concentration $(n=5$, mean \pm SEM), and platelet counts $(n=6$, mean \pm SEM) were significantly lower, while the counts of monocytes (Mono) $\left(n=5\right.$, mean \pm SEM), neutrophils (Neutro) $\left(n=5\right.$, mean \pm SEM) and eosinophils (Eosino) $\left(n=5\right.$, mean \pm SEM) were significantly elevated in S/c29a3 ${ }^{-/-}$

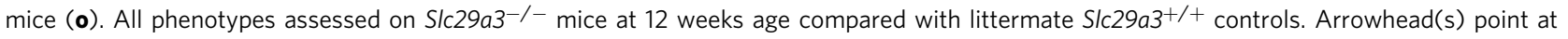
abnormalities. Statistical analyses were performed by ANOVA with Tukey's multiple comparisons post-test and two-tailed Student's $t$-test. ${ }^{\star} P<0.05$. blue

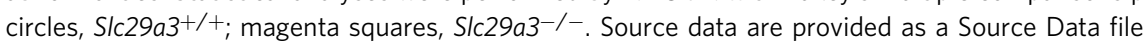

observed that Slc29a3 ${ }^{-1-}$ HSCs, and not Slc29a3 ${ }^{+1+}$ HSCs, failed to reconstitute the bone marrow and thus were unable to rescue the survival of the lethally irradiated mice (Fig. $3 g$, h). Importantly, when Slc29a3-1- HSCs ectopically expressing ENT3 were transplanted into lethally irradiated mice, a dramatic improvement in survival over nontransplanted mice was observed (Fig. 3i-k). In addition, the transplantation of Slc29a3 ${ }^{-1-}$ MSCs ectopically expressing ENT3 moderately extended survival (Fig. 3i-k). Consistently, combined transplantation of Slc29a3 $3^{-1-}$ HSCs overexpressing ENT3 and Slc29a3 $3^{-1}$ MSCs overexpressing ENT3 (stem cell transplant; SCT) showed the highest increase in survivability and bone marrow reconstitution (Fig. 3i-k). No survival benefits were observed in mice transplanted with RFP-overexpressing Slc29a3-1- MSCs or RFP-overexpressing Slc29a3 ${ }^{-1-}$ HSCs (Fig. 3i-k). These data further supported the hypothesis that ENT3-dependent stem cell mechanisms are primarily involved in bone marrow exhaustion.

As MSCs and HSCs that jointly share the same microenvironmental niche are both affected in their differentiation functions (Fig. 2a-f), it is possible that the alterations in one cell type are secondary to changes in paracrine factors derived from the other and/or common microenvironmental niche. Therefore, we sought to assess whether the influence of ENT3 on HSCs is altered according to the bone marrow microenvironment to which they are provided access for reconstitution. To eliminate the basal HSCs from Slc29a3 $3^{+/+}$and Slc29a3 $3^{-/-}$bone marrow, we lethally irradiated the mice that were subsequently subjected to HSC transplantation performed using the opposite donor-recipient combination. The failure of hematopoietic reconstitution in irradiated WT mice (that contains Slc29a3 $3^{+/+}$niche) by Slc29a3 $3^{-1-}$ HSC and rescue of bone marrow failure in irradiated

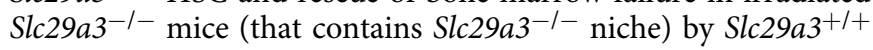
HSCs suggested that ENT3 affected stem cell functions in a cellautonomous manner (Supplementary Fig. 4b).

An autophagy defect underlies stem cell deficits. As autophagy plays obligate roles in stem cell regulation ${ }^{31}$ and is inextricably linked to lysosomes ${ }^{32,33}$, defective nucleoside transport by ENT3 across lysosomal membranes might impair autophagic regulation of stem cells. Consistent with the hypothesis, basal autophagy was reduced in Slc29a3 ${ }^{-1-}$ MSCs and HSCs as evidenced by the decreased appearance of the autophagosome marker LC3-II and accumulation of the autophagy substrate Sequestosome1/p62 34,35 (Fig. 4a). This defect was more evident when cells were deprived of glucose (Fig. 4a, b), indicating that ENT3 regulates both basal and starvation-induced autophagy. The reduction in LC3-II accumulation was more dramatically visualized when autophagic flux was inhibited with bafilomycin $\mathrm{A}_{1}$ (an inhibitor of vacuolar-type $\mathrm{H}^{+}$-ATPase) under glucose-starved conditions (Fig. 4a, b). TEM analysis revealed fewer autophagosomes and an increased number of lysosomes in Slc29a3-1- MSCs compared to MSCs isolated from WT littermate controls (Fig. 4c). In addition, Slc29a3-1- MSCs showed an increased presence of mitochondria and ER (Fig. 4c), suggesting a possible lack of autophagic removal of these organelles ${ }^{36-38}$. Following glucose deprivation, loss of ENT3 was associated with compromised cellular viability in both MSCs and HSCs, which could be acutely rescued via activation of the autophagy response with rapamycin ${ }^{39}$, overexpression of the autophagy-related gene ATG $7^{40}$ or reconstitution of the system with an ENT3 expression plasmid (Fig. 4d-f). Caspase activity measurements indicated that the cell death program was minimally involved in the rapamycininduced recovery of Slc29a3-1- cells (Fig. 4d). Moreover, when Slc29a3-1- MSCs were induced to differentiate into osteoblasts in the presence of rapamycin or ATG7 overexpression, both the expression of osteoblast transcription factor and marker genes were rescued (Fig. 4g). Taken together, these data suggest that autophagic deficits contribute to stem cell defects in an ENT3deficient state.

The differentiation of adult stem cells and autophagy are interdependent mechanisms ${ }^{41}$. When stem cells differentiate down a lineage, they reduce their dependence on autophagy, and, conversely, when autophagy is defective, stem cell functions are compromised $^{42}$. We next investigated whether ENT3 could modulate autophagy in a non-stem cell (HEK293) line. Silencing ENT3 (Supplementary Fig. 5a-c) compromised autophagy in HEK293 cells as well, resulting in reduced LC3-II levels (Supplementary Fig. 5d). Importantly, this autophagic regulation appears to be specific to ENT3. Overexpression of ENT3, but not ENT1, and to a lesser extent ENT2, was associated with increased LC3-puncta and increased processing of $\mathrm{LC}^{35}$ (Supplementary Fig. 5e-g). Again, reduced LC-3 II accumulation in ENT3silenced cells and increased LC-3 II accumulation in ENT3overexpressing cells were more evident when autophagic flux was inhibited with bafilomycin A1 (Supplementary Fig. 5d, e). To further define the nature of the autophagic regulation by ENT3, we analyzed flux through the pathway using the tandem tagged LC3 (RFP-GFP-LC3) construct. The GFP signal is quenched in the acidic environment of the lysosome, while the red signal is maintained, allowing rapid differentiation of autophagosomes (yellow puncta) and autolysosomes (singly RFP + puncta) by fluorescence ${ }^{35}$. ENT3 increased singly-positive RFP LC-3 puncta fluorescence (Supplementary Fig. 5h, i). When autophagic flux was induced with rapamycin in the presence of chloroquine (to prevent lysosomal degradation), ENT3-silenced HEK293 cells, but not ENT3-reconstituted cells, showed decreased LC3-II (Supplementary Fig. 5j). Altogether, these results identified a direct role for ENT3 in autophagy induction and further 

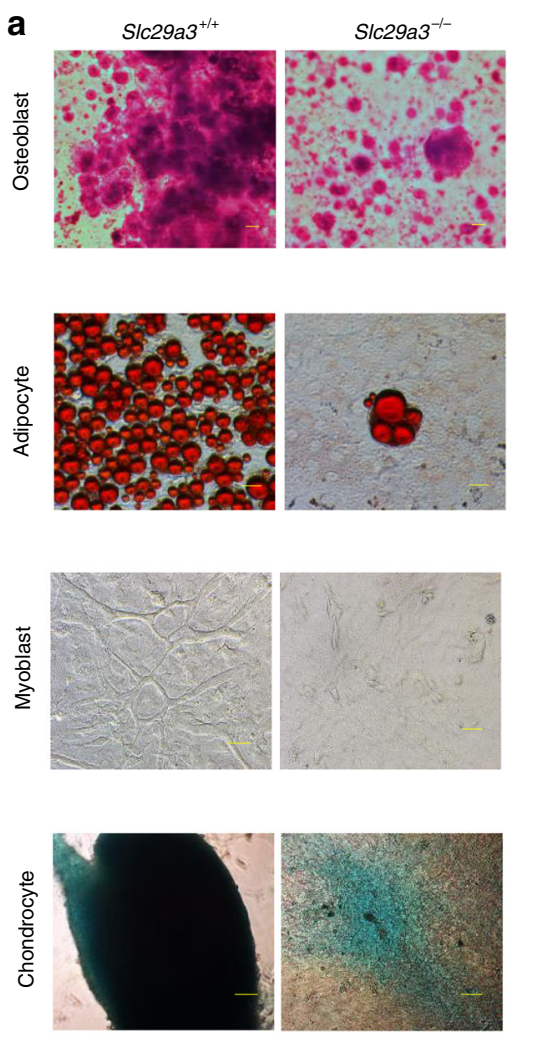

C
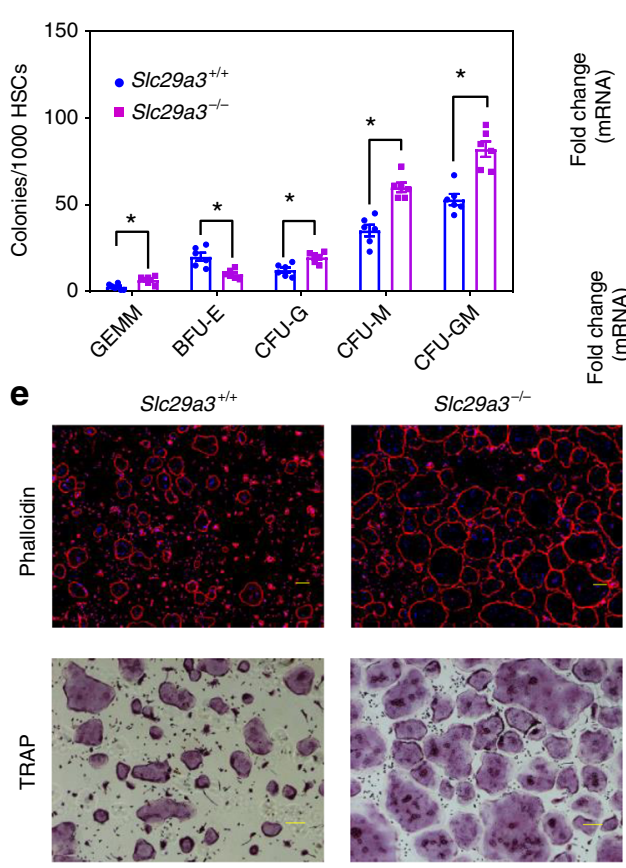

b

d
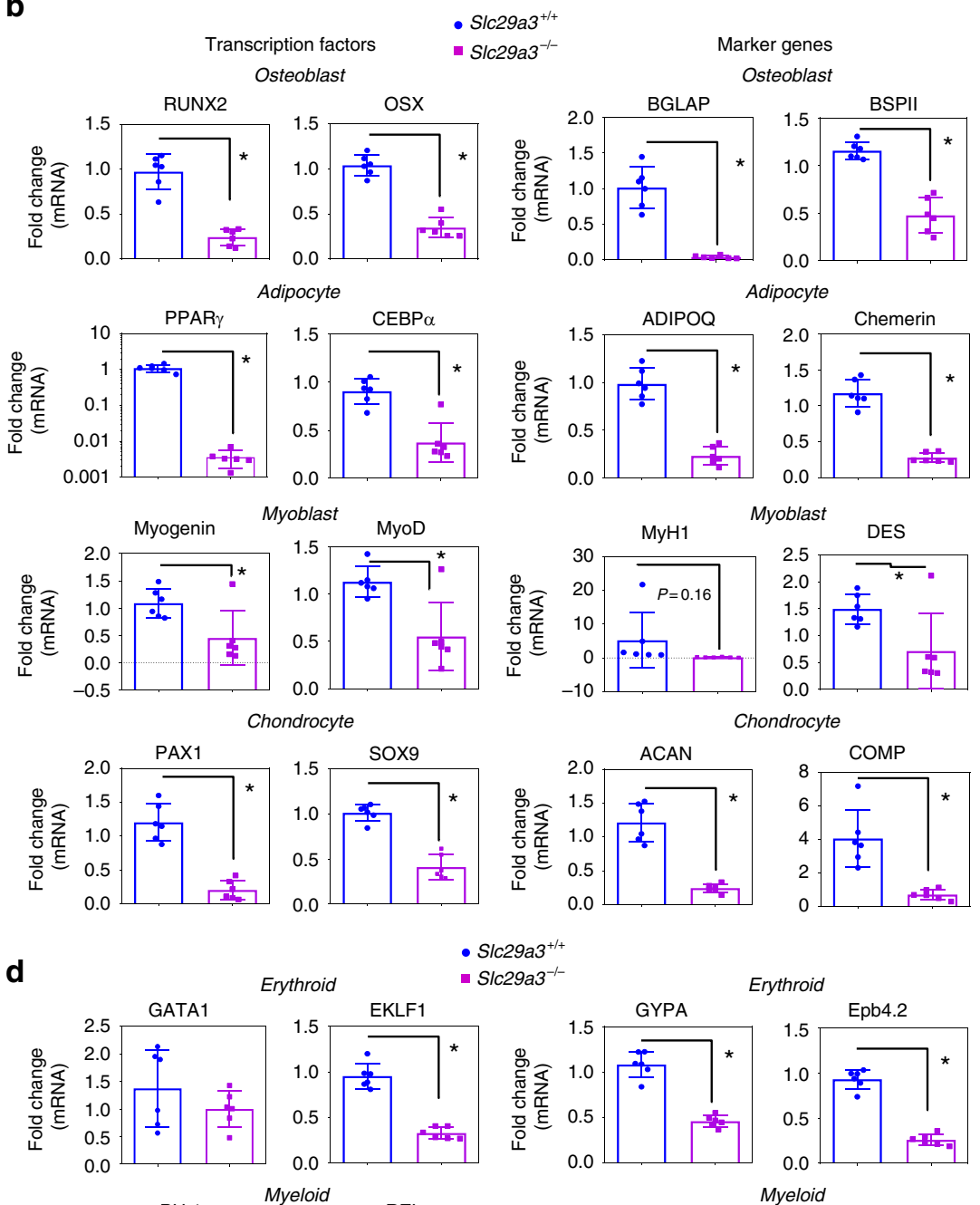

$S / c 29 a 3^{+/+}$
SIc29a3

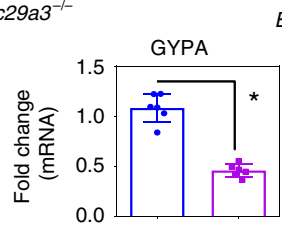

Erythroid
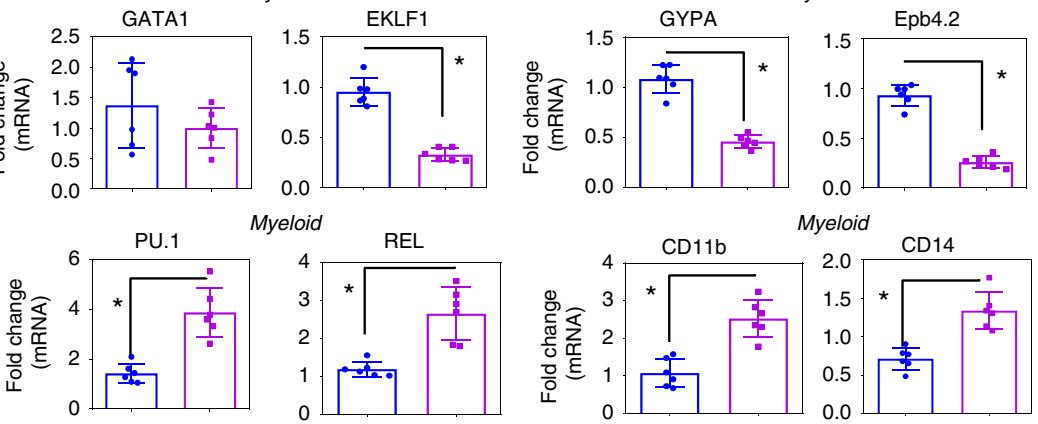

Myeloid

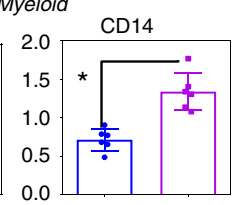

Fig. 2 ENT3 is essential for stem cell differentiation. MSC differentiation into osteoblasts (mineralized nodules; pink), adipocytes (lipid droplets; red), myoblasts (myotube formation), and chondrocytes (cartilage nodule; bluish green). Scale bar: $100 \mu \mathrm{m}(\mathbf{a})$. mRNA expression of transcription factors and markers after 14 (osteoblasts and adipocytes) and 28 (myoblasts and chondrocytes) days of MSC differentiation $(n=6$, mean \pm SEM) (b). HSC colonyforming cell assay $(n=6$, mean \pm SEM) (c) and transcript expression after 14 days of differentiation $(n=6$, mean \pm SEM) (d). Osteoclast formation (actin ring; red, above) (Scale bar: $100 \mu \mathrm{m})(\mathbf{e})$ and TRAP (tartrate-resistant acid phosphatase) expression (purple; below) (Scale bar: $100 \mu \mathrm{m})(\mathbf{e})$ and secretion with HSC differentiation ( $n=6$, mean \pm SEM) (f). GEMM, granulocyte, erythrocyte, monocyte, megakaryocyte; BFU-E, burst-forming unit-erythroid; CFU, colony-forming unit; CFU-M, CFU-macrophage; CFU-G, CFU-granulocyte; CFU-GM, CFU-granulocyte/macrophage. Statistical analyses were performed using ANOVA with Tukey's multiple comparisons post-test and two-tailed Student's $t$-test. ${ }^{\star} P<0.05$. Source data are provided as a Source Data file 
a

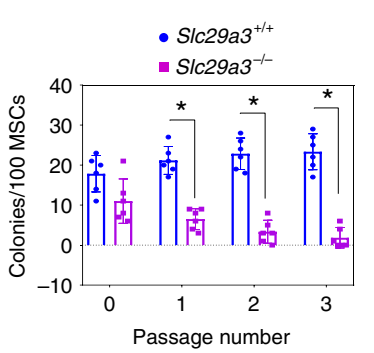

e

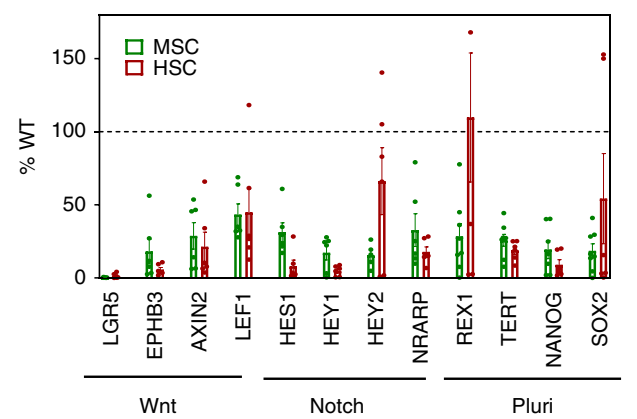

g

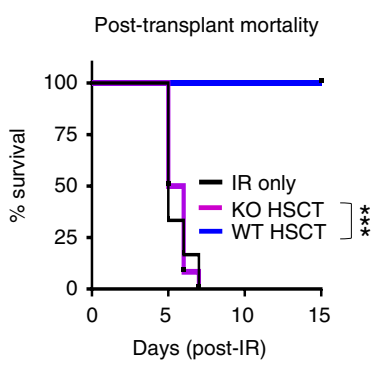

h

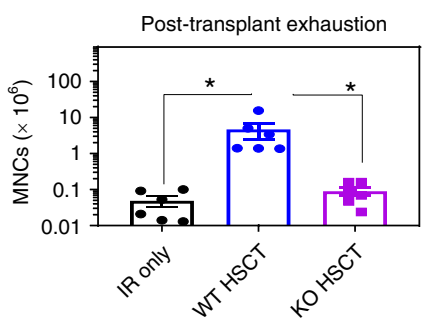

b $\quad .4 c_{29} 3^{+++} \quad$ C
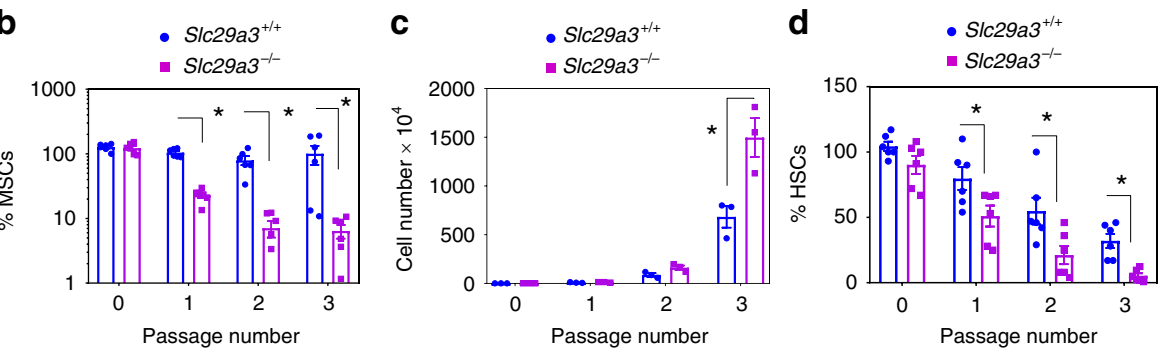

f

Primary exhaustion
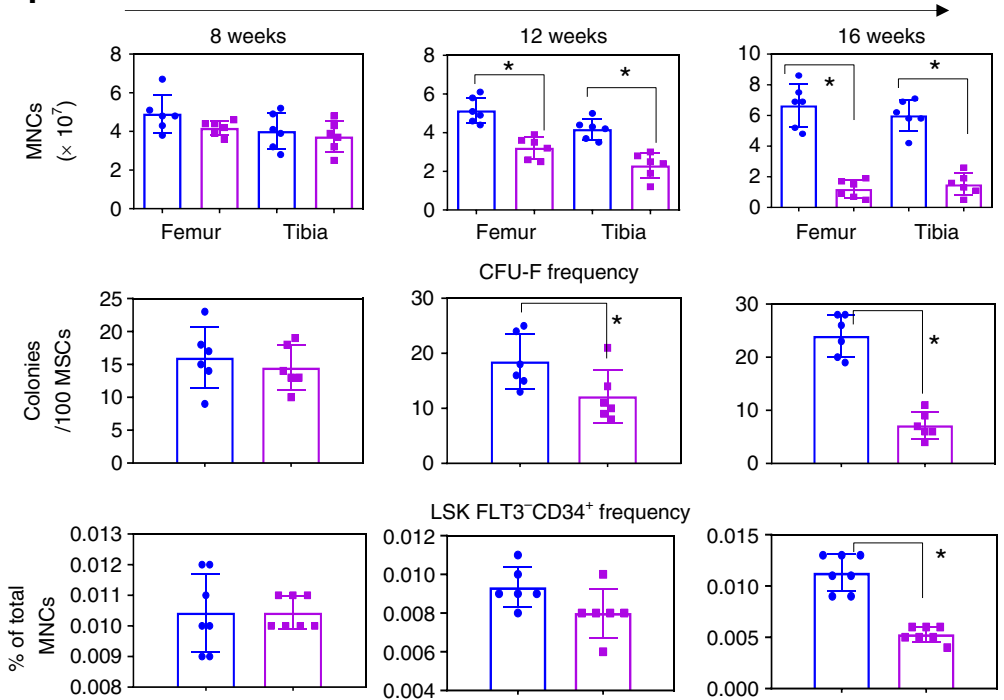

i

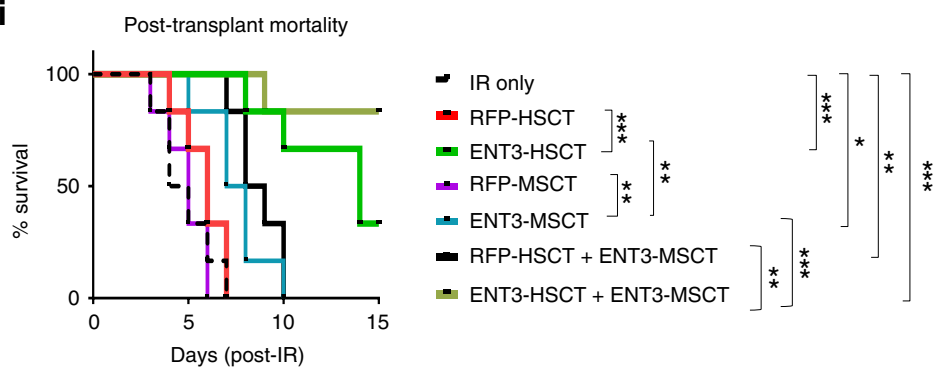

j
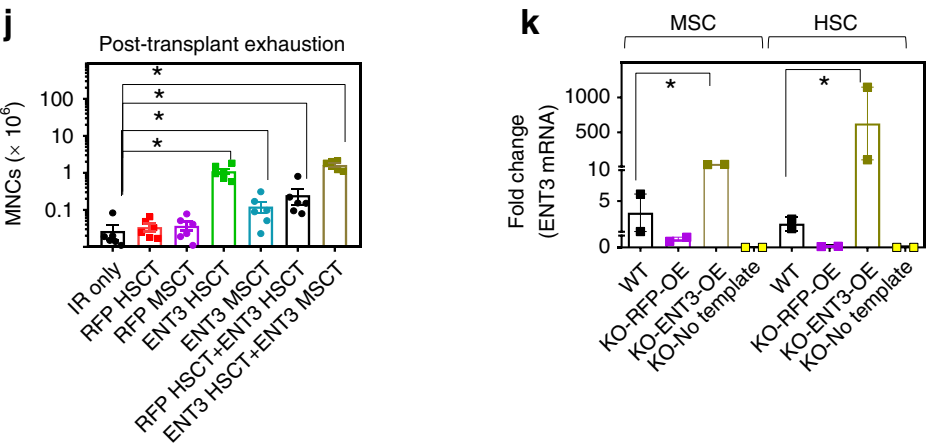

supported the hypothesis that ENT3 regulation of stem cell proliferation and differentiation occurs via autophagic modulation.

ENT3 regulates autophagy via the AMPK-mTOR-ULK pathway. As a high-capacity adenosine transporter ${ }^{20,22,23}$, whether ENT3 influences adenosine monophosphate kinase (AMPK) activity to perturb autophagy has been unexplored. Interestingly,
Slc29a3 ${ }^{-1-}$ MSCs and HSCs showed impaired activation of AMPK (measured via phosphorylation on Thr172 on AMPK) in response to glucose deprivation (Fig. 5a) or AICAR (an inducer of AMPK activity) treatment (Fig. 5a). Reduced phosphorylation of the AMPK target, acetyl CoA carboxylase (ACC $)^{43}$ in Slc29a3-1MSCs and HSCs (Fig. 5a) and a loss of AMPK phosphorylation in ENT3 knockdown HEK293 cells (HEK293KD) (Fig. 5b) further suggested a relationship between ENT3 expression and AMPK activity. Reconstitution of ENT3 in HEK293KD cells resulted in 
Fig. 3 ENT3 is essential for self-renewal. MSC clonogenicity when subjected to serial passage $(n=6$, mean \pm SEM) (a). Percent cells expressing MSC markers after each passage of serial cloning $(n=6$, mean \pm SEM) (b). Culture expansion capacity of HSCs $(n=3$, mean \pm SEM) (c). Percent of cells expressing HSC markers after each passage $(n=6$, mean \pm SEM) (d). Expression of Wnt and Notch targets and pluripotency (pluri) marker genes in Slc29a3-1- MSCs and HSCs ( $n=6$, mean \pm SEM) (e). HSCs and MSCs were derived from 12-weeks-old animals (a-e). Cellularity ( $n=6$, mean \pm SEM), MSC CFU $-\mathrm{F}\left(n=6\right.$, mean \pm SEM) and LSK FLT3- ${ }^{-} \mathrm{CD}^{+}{ }^{+}(n=7$, mean \pm SEM) frequencies in bone marrow measured in different age groups () . MNC, mononuclear cells; CFU-F, CFU-Fibroblast $(\mathbf{f})$. Ability of HSCs $\left(1 \times 10^{4}\right.$ cells $)$ to rescue radiation lethality in S/c29a3 ${ }^{-/-}$mice after transplantation presented as Kaplan-Meier survival curves $\left(n=6 /\right.$ group, ${ }^{\star \star \star} P<0.001$; Mantel-Cox test) (g) and post transplantation bone marrow cellularity $(n=6$, mean \pm SEM)

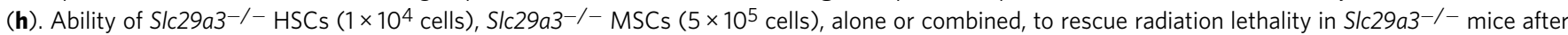
the expression of RFP or ENT3 presented as Kaplan-Meier survival curves, $n=6 /$ group, ${ }^{\star} P<0.05,{ }^{\star \star} P<0.01,{ }^{\star \star \star} P<0.001$; Mantel-Cox test (i) and post transplantion bone marrow cellularity $\left(n=6 /\right.$ group, ${ }^{\star} P<0.05$; Mantel-Cox test) (j). Relative expression of ENT3 in MSCs and HSCs transduced with a lentiviral construct harboring RFP or ENT3 compared with expression in WT mice (k). HSCs and MSCs were derived from 8-weeks-old animals (g-k). Statistical analyses were performed using ANOVA with Tukey's multiple comparisons post-test and two-tailed Student's $t$-test. ${ }^{*} P<0.05$. Source data are provided as a Source Data file. blue circles, S/c29a3+/+; magenta squares, SIc29a3-/-

more robust AMPK phosphorylation than observed in parental cells, suggesting an additive effect of ENT3 and glucose deprivation in regulating AMPK phosphorylation (Fig. 5b, c). Intriguingly, when glucose-deprived Slc29a3-1- HSCs and MSCs were treated with increasing concentrations of AICAR, there was a near-complete rescue of cellular viability with marked induction of MSC differentiation into osteoblasts (Fig. 5d, e), an effect even more pronounced than that seen with rapamycin (mTOR inhibitor ${ }^{44}$; (Fig. 4d, g)). When investigated for possible AMPKmTOR interplay, Slc29a3 $3^{-1-}$ MSCs and HSCs, as well as HEK293KD cells, had constitutively high phospho-mTOR $(\mathrm{S} 2448)^{45}$ that was sustained even under glucose deprivation, AICAR treatment or rapamycin treatment (Fig. 5a, b). Phosphorylation of downstream mTOR targets phospho-S6K (T389) 46 and phospho-4EBP1 (T37/46), ${ }^{47}$ remained relatively high in Slc29a3-l- HSCs, MSCs and HEK293KD cells (Fig. 5a, b). When analyzed for the differential phosphorylation of the autophagyinitiating kinase, ULK1 (Unc-51 like Autophagy Activating Kinase 1$)^{48}$, the levels of phosphoULK1-S757 and phosphoULK1S555 (the mTOR and AMPK phosphorylation sites, respectively) were reciprocally regulated such that ULK1 phosphorylation at the mTOR site (S757; that inhibits autophagy) was sustained at a higher level while that at the AMPK site (S555; that activates autophagy) was reduced (Fig. 5a, b). Treatment with AICAR marginally restored the levels of ULK1 phosphorylation at S555 in Slc29a3-/- MSCs and HSCs although Slc29a3-1MSCs required a higher concentration of $\operatorname{AICAR}(500 \mu \mathrm{M})$ than Slc29a3 $3^{-l-}$ HSCs $(100 \mu \mathrm{M})$ to produce the same effect (Fig. 5a and Supplementary Fig. 5k). In addition, AICAR induced LC3-II levels in both Slc29a3-1- MSCs and HSCs albeit with lesser magnitudes than that observed in WT cells (Fig. 5a, b and Supplementary Fig. 5k). Other AMPK activators such as metformin and azacytidine also moderately induced LC3-II levels in Slc29a3-I- MSCs (Supplementary Fig. 5l). Furthermore, ENT3 expression synergistically enhanced the effects of the mTOR inhibitor, Torin 1, to induce autophagy (Supplementary Fig. $5 \mathrm{~m}$ ). In addition to glucose stimulation, amino acid stimulation, to a lesser degree, increased mTOR phosphorylation, suggesting that mTORC1 regulation by ENT3, might not be exclusively due to AMPK activity (Supplementary Fig. 6a).

As both AMPK and mTOR have been shown to be regulated on the lysosome ${ }^{46,48}$, we next interrogated whether the subcellular distribution of these kinases differed in Slc29a3 ${ }^{-1-}$ MSCs. While AMPK displayed a diffuse, cytosolic staining pattern with the occasional presence of large dotted structures, mTOR showed a predominantly vesicular staining pattern throughout the cytoplasm (Fig. 5f). However, unlike the reduced phospho-AMPK levels observed in total cell lysates prepared from Slc29a3 ${ }^{-1-}$ MSCs (Fig. 5a), the phospho-AMPK levels were not reduced in the lysosomal fractions isolated from Slc29a3 ${ }^{-1-}$
MSCs (Fig. 5g). These results suggested that the AMPK regulatory events predominantly occurred in the non-lysosomal (i.e., cytosolic) compartments of Slc29a3 ${ }^{-1-}$ MSCs. On the other hand, the phospho-mTOR levels (S2448) were relatively higher in the lysosomal fractions isolated from Slc29a3-1- MSCs (Fig. 5g), suggesting that mTOR regulation, at least in part, occurred on the lysosome. These results were also consistent with the increased proliferation of lysosomes observed in Slc29a3-1- MSCs (Fig. 4c). Exogenous ENT3 expression in AMPK a1/a2 double KO mouse embryo fibroblasts (MEFs) ${ }^{49}$ failed to generate an adequate autophagic response with reduced LC3 II formation, which corroborated an integral role for AMPK signaling in eliciting ENT3's autophagic responses (Fig. 5h).

As ENT3 is an acidic pH-activated transporter ${ }^{19,22,23}$ and the acidic milieu of eukaryotic lysosomes can generate high levels of adenosine through the degradation of nucleic acids arising from autophagic (or phagocytic) pathways, we next examined whether ENT3-mediated translocation of a lysosomal pool of adenosine into cytosol activates AMPK signaling (Fig. 6a). Supportively, cell fractionation and mass spectrometry analysis of lysosomal fractions of $S l c 29 a 3^{-1-}$ spleen (derived from mesodermal mesenchyme and harbors HSCs) and HEK293KD cells demonstrated the increased accumulation of adenosine (Fig. $6 \mathrm{~b}$ and Supplementary Fig. 6). These findings indicated that reduced lysosomal recycling of adenosine negatively influences AMPK signaling in Slc29a3 ${ }^{-1-}$ mice. To test directly, we tracked the efflux of adenosine from lysosomes and measured the changes in cytosolic adenosine, AMP and AMPK phosphorylation after the transduction of HEK293KD with increasing multiplicity of infection (MOI) of hENT3-harboring retroviruses. An increase in the lysosomal efflux of adenosine with parallel increases in AMP and AMPK phosphorylation levels and LC3 lipidation as well as a decrease in AMPK downstream effectors (pmTOR) was observed in a saturable manner with a graded increase in ENT3 expression (Fig. 6c, d). In addition to the accumulation of endogenously generated adenosine in lysosomes, a relatively higher accumulation of AICAR (an adenosine analog transported by ENT3 (Fig. 6e)) was also observed in the lysosomal fractions isolated from AICAR-treated Slc29a3-1- MSCs (Fig. 6f), partly explaining the attenuated response of AICAR to activate AMPK in Slc29a3 $3^{-1-}$ MSCs (Fig. 5a). Moreover, the activation of AMPK by adenosine was dependent on its conversion to AMP because the inhibition of adenosine kinase by iodotubericidin completely abolished ENT3-induced AMPK phosphorylation (Fig. 6g). Furthermore, the capacity of ENT3 to increase AMPK phosphorylation was dependent on its lysosomal localization because the overexpression of the $\mathrm{N}$-terminal truncated mutant $(\Delta \mathrm{N} 36$ ENT3-YFP) that removes the lysosomal-targeting signal from $\mathrm{ENT}^{20}$ failed to induce AMPK phosphorylation in HEK293KD cells (Fig. 6h, i). 


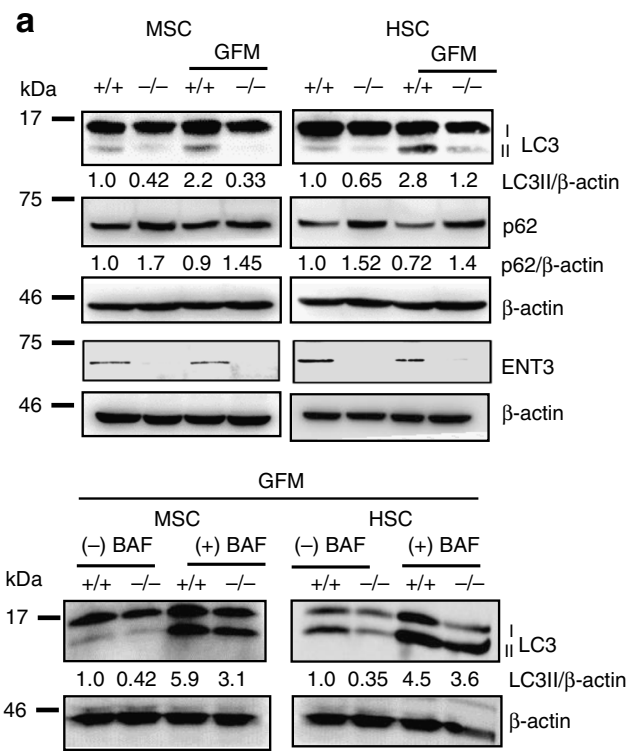

b

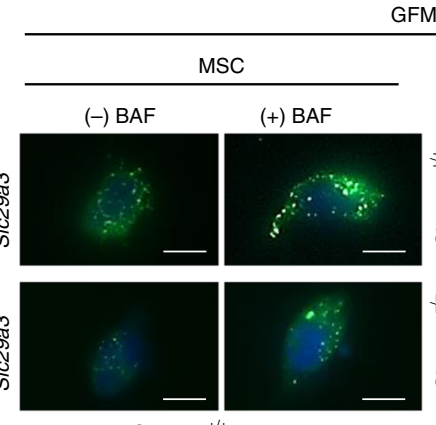

GFM
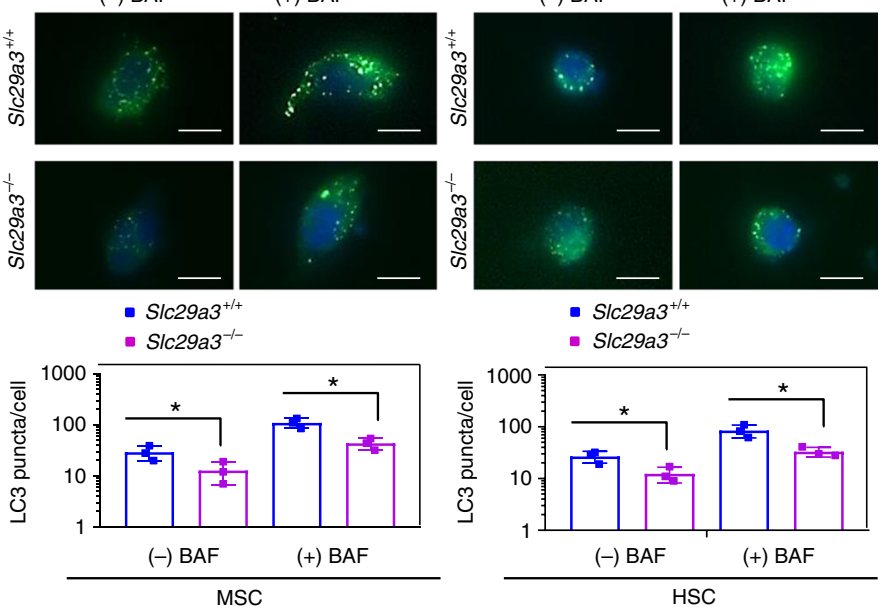

(-) BAF

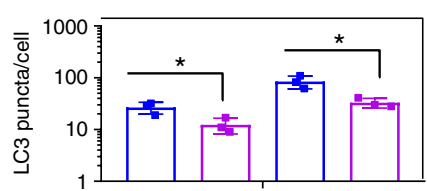

(-) BAF

(+) BAF

c

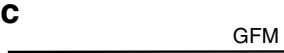

d
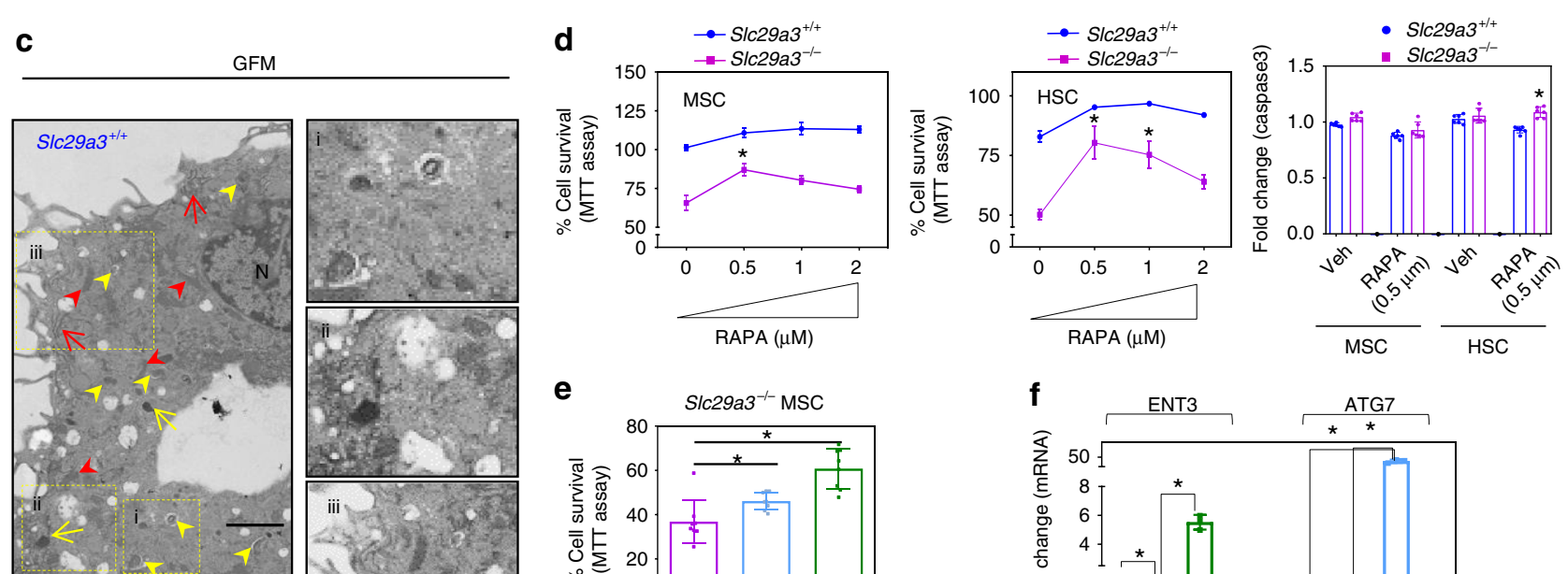

e
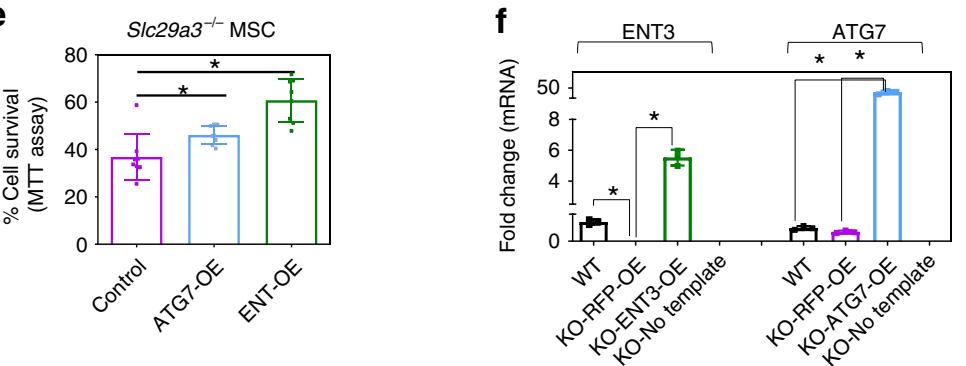

g
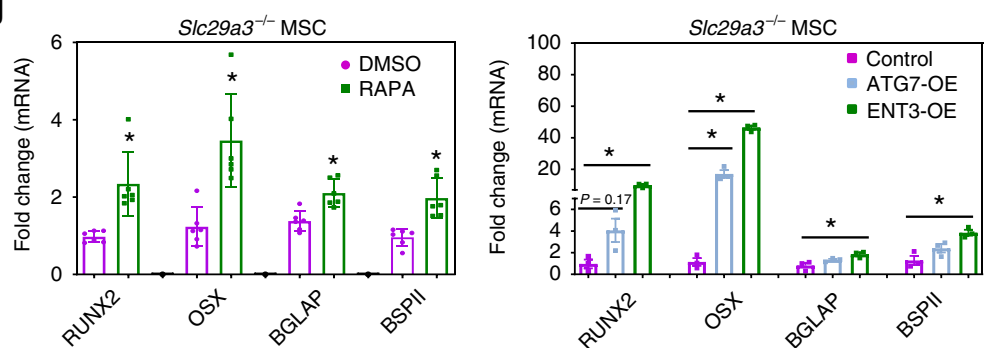

ENT3 loss alters fatty acid utilization and bioenergetics. In the next set of experiments, we tested whether the autophagy defect observed in cultured MSCs and HSCs was also evident in mouse tissues. Examination of several organs from adult Slc29a3-1mice demonstrated the increased accumulation of p62 (Fig. 7a). In addition, Slc29a3 ${ }^{-1-}$ spleens showed alterations in AMPK and downstream targets (Fig. 7b). Reduced levels of ATG7 (Fig. 7b), but not transcription factor EB (TFEB) (Fig. 7c), further suggested the involvement of the core autophagic machinery but not lysosome-to-nucleus signaling ${ }^{32,35}$. Consistently, TEM analysis of Slc29a3 ${ }^{-I-}$ mouse livers demonstrated fewer autophagosomes and more lysosomes, mitochondria, and ER (Fig. 7d).
Intriguingly, Slc29a3 $3^{-/-}$livers displayed fewer and smaller lipid droplets (Supplementary Fig. 7a). In addition, BODIPY 493/ 503 staining of neutral lipid droplets was lower in oleic acidtreated Slc29a3 $1-$ hepatocytes, indicating defects in the formation of lipid droplets (Supplementary Fig. 7b). These observations, along with the perturbation in the major energy and nutrient-sensing pathways (AMPK and mTOR), suggested potential metabolic alterations additionally driving ENT3-related dysfunction in mice. As the formation of neutral lipid droplets largely relies on glucose and patients with PHID syndrome exhibit insulin-dependent diabetes mellitus ${ }^{6}$, we tested whether Slc29a3 ${ }^{-1-}$ mice display an abnormal glycemic profile. Although 
Fig. 4 ENT3 loss impairs induction of autophagic response. Representative immunoblots of LC3 and p62 protein expression in MSCs and HSCs under glucose starvation ( $1 \mathrm{~h}$; above) followed by treatment with vehicle ((-) BAF) or bafilomycin A1 (( +)BAF; $100 \mathrm{nM}, 4 \mathrm{~h})$ under glucose starvation (below). $\beta$-Actin served as the loading control (a). Representative fluorescent images (above) and quantification (below) of LC3 puncta formation (green) in MSCs and HSCs under glucose starvation $(1 \mathrm{~h})$ followed by treatment with vehicle $((-) \mathrm{BAF})$ or bafilomycin $\mathrm{A} 1((+) \mathrm{BAF} ; 100 \mathrm{nM}, 4 \mathrm{~h})$ under glucose starvation. Original magnification, $\times 60$; Scale bar: $10 \mu \mathrm{m}$. Nuclei stained with DAPI. $(n=3$, mean \pm SEM) (b). TEM analysis of autophagosome (yellow arrowhead) and

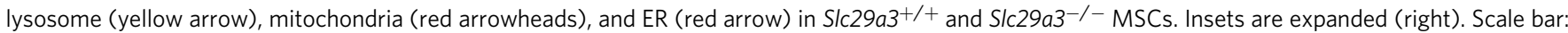
$2 \mu \mathrm{m}$. N, nucleus (c). Effect of RAPA (rapamycin) treatment $(0.5-2 \mu \mathrm{M})$ on GFM-induced MSC and HSC survival $(n=4$, mean \pm SEM) and death $(n=6$, mean \pm SEM) as assayed by MTT assay and active caspase 3 measurement, respectively. The ' $0^{\prime \prime}$ concentration compares S/c29a3-/- cell survival with

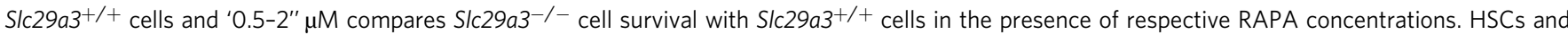
MSCs were derived from 12-weeks-old mice (d). Effect of ATG7 (ATG7-OE) or ENT3 (ENT3-OE) overexpression on glucose starvation-induced MSC survival $(n=8$, mean \pm SEM) (e). Relative expression of ENT3 and ATG7 in Slc29a3-/- MSCs and HSCs transduced with lentiviruses harboring RFP or ENT3 compared with expression in S/c29a3 $+/+(W T)$ mice $(n=3$, mean \pm SEM) (f). Effect of RAPA (0.5 $\mu$ M), ATG7-OE or ENT3-OE on osteogenic medium (3 days)-induced transcription factor and marker gene expression in S/c29a3-/- MSCs $(n=6$, mean \pm SEM) (g). Statistical analyses were performed using ANOVA with Tukey's multiple comparisons post-test and two-tailed Student's $t$-test. ${ }^{\star} P<0.05$. All phenotypes were assessed in MSCs and HSCs derived from 12-week-old mice. GFM, glucose-free medium. Source data are provided as a Source Data file

the intraperitoneal glucose tolerance test revealed a mildly elevated glycemic response in Slc29a3 $3^{-1-}$ mice, the extent of glycemia was minimal (Supplementary Fig. 7c). Therefore, to gain a better understanding of the metabolic dysregulation in Slc29a3 $3^{-1-}$ cells, we performed mass spectrometry-based untargeted metabolomic profiling of the Slc29a3 ${ }^{-1-}$ mouse liver. As stem cells (particularly HSCs) were sample limiting for in-depth metabolomic analyses, we chose liver tissue for this analysis primarily because of its high responsiveness to autophagy and its role in regulating systemic metabolic homeostasis. Additionally, the liver has the highest ENT3 expression among all mouse tissues (http://symatlas.gnf.org). This analysis detected 8410 total metabolites in the Slc29a3 ${ }^{-1-}$ liver, among which 879 metabolites were significantly altered $(P<0.05$ and fold change $>2.0)$ (Fig. 8a, Supplementary Fig. 7d, and Supplementary Data 1). A subsequent VIP plot and S-plot generated from supervised PLS-DA and OPLS-DA analysis, respectively, and mass spectral confirmation of second-order MS fragmentation patterns identified lipids as the most affected superclass (95 out of 175; Fig. 8a and Supplementary Fig. 7e, f and 8). To delineate alterations within the lipid superclass, we subsequently conducted mass spectrometry-based targeted lipidomics. This analysis revealed that 120 of 498 individual lipids examined were significantly altered $(P<0.05$ and fold change $>2.0$ ) (Fig. 8b, Supplementary Fig. 9, and Supplementary Data 2). Remarkably, highly elevated polyunsaturated and monounsaturated free fatty acids and generalized decreases in neutral lipids (fatty acylglycerol esters (changes in triglycerides $>$ diglycerides $>$ monoglycerides) and cholesteryl esters), prenol lipids, glycerophospholipids, and steroids were identified as striking features (Fig. 8b and Supplementary Fig. 9). Consistently, Slc29a3 $3^{-1-}$ mouse plasma displayed severe dyslipidemic features, including hypocholesterolemia, hypotriglyceridemia, and hypolipoproteinemia (Supplementary Fig. 10a).

As metabolic plasticity is a critical requirement for stem cell lineage specification, we next investigated whether metabolic reprogramming also occurred in Slc29a3-1- MSCs. Although the extracellular acidification rate (a measure of glycolysis) was unaltered (Fig. 8c), a Seahorse XF96 cell Mito test demonstrated that the mitochondrial oxygen consumption rate was reduced in Slc29a3-1- MSCs under bioenergetics stress (Fig. 8d). In addition, decreased maximal respiration and ATP production, increased non-mitochondrial respiration and proton leak, and a loss of the spare reserve capacity were identified in Slc29a3-1MSCs (Fig. 8c-e). Importantly, when measured for endogenous and exogenous palmitic acid oxidation, simultaneous reductions in mitochondrial $\beta$-oxidation capacities were identified from both sources (Fig. 8f). Furthermore, targeted mass spectrometry analysis of select lipid metabolites recapitulated the elevated presence of several free fatty acids and the decreased presence of diglycerides, triglycerides, phosphatidylcholine, and phosphatidylethanolamine in Slc29a3-1- MSCs (Supplementary Fig. 10b) similar to that observed in the mouse liver. Compared with WT MSCs, Slc29a3 $3^{-1-}$ MSCs displayed a relatively quiescent and glycolytic phenotype (Fig. 8g), indicating metabolic reprogramming and alterations in fuel source selection. Treatment of Slc29a3 $1-$ MSCs with AICAR partially normalized lipid metabolite alterations with the restoration of several metabolites to levels comparable to those of WT (Supplementary Fig. 10b). As AMPK also restored proliferation and differentiation defects in Slc29a3 ${ }^{-1-}$ MSCs (Fig. 5d, e), taken together, these findings suggested mitochondrial bioenergetics alterations can additionally propel stem cell deficits in Slc29a3 $3^{-1-}$ mice.

Rescue of ENT3-related dysfunctions in KO mice. Finally, we evaluated whether stem cell transplantation (SCT, which increases short-term survival in lethally irradiated mice (Fig. 3i)) or in vivo AMPK activation (AICAR treatment) can reduce ENT3 disease pathologies and increase the long-term survival of Slc29a3 ${ }^{-1-}$ mice. We injected $1 \times 10^{4}$ Slc29a3 ${ }^{+1+}$ HSCs and $5 \times$ $10^{5}$ MSCs (once) intravenously into 10-week-old asymptomatic Slc29a3-1- mice (SCT) or AICAR at $50 \mathrm{mg} / \mathrm{kg}$ (daily) by intraperitoneal delivery beginning at 10 weeks of age (Fig. 9a). While saline-treated Slc29a3 ${ }^{-1-}$ mice showed a rapid decline in survival as expected, the chronic administration of AICAR significantly prevented morbidity and improved the overall survival in Slc29a3 $3^{-1-}$ mice with $\sim 50 \%$ of mice surviving beyond 22 weeks (Fig. 9a). Despite the patchy alopecia observed in facial regions particularly near the muzzle and surrounding the eyes (Fig. 9b), the AICAR-treated surviving mice at 28 weeks of age (after $\sim 10$ weeks of death of saline-treated Slc29a3-/- mice) showed overall improvement in health, body weight and hematopoiesis compared with Slc29a3 $3^{-1-}$ mice at 12 weeks of age (Fig. 9a-c). Strikingly, SCT mice showed $100 \%$ survival at 28 weeks of age with notable improvement in health, body weight and hematopoiesis compared with Slc29a3-1- mice at 12 weeks of age (Fig. 9a-c). EchoMRI and necropsy revealed in both AICAR-treated and SCT mice with preserved muscle mass, but only SCT mice showed preserved adipose tissue mass (Fig. 9d-f). When the capacity of bone marrow MSCs were analyzed, MSCs derived from SCT mice, but not AICAR-treated mice, fully restored the colony-forming unit-fibroblast (CFU-F) to WT levels (Fig. 9g). To further confirm whether the CFU-F-derived progenies were truly MSCs, we assessed MSC surface marker expression by flow cytometry. The percentage of MSCs within the CFU-F at each passage was markedly higher in the SCT group (than KO group), confirming the recovery of MSC self-renewal in 
a

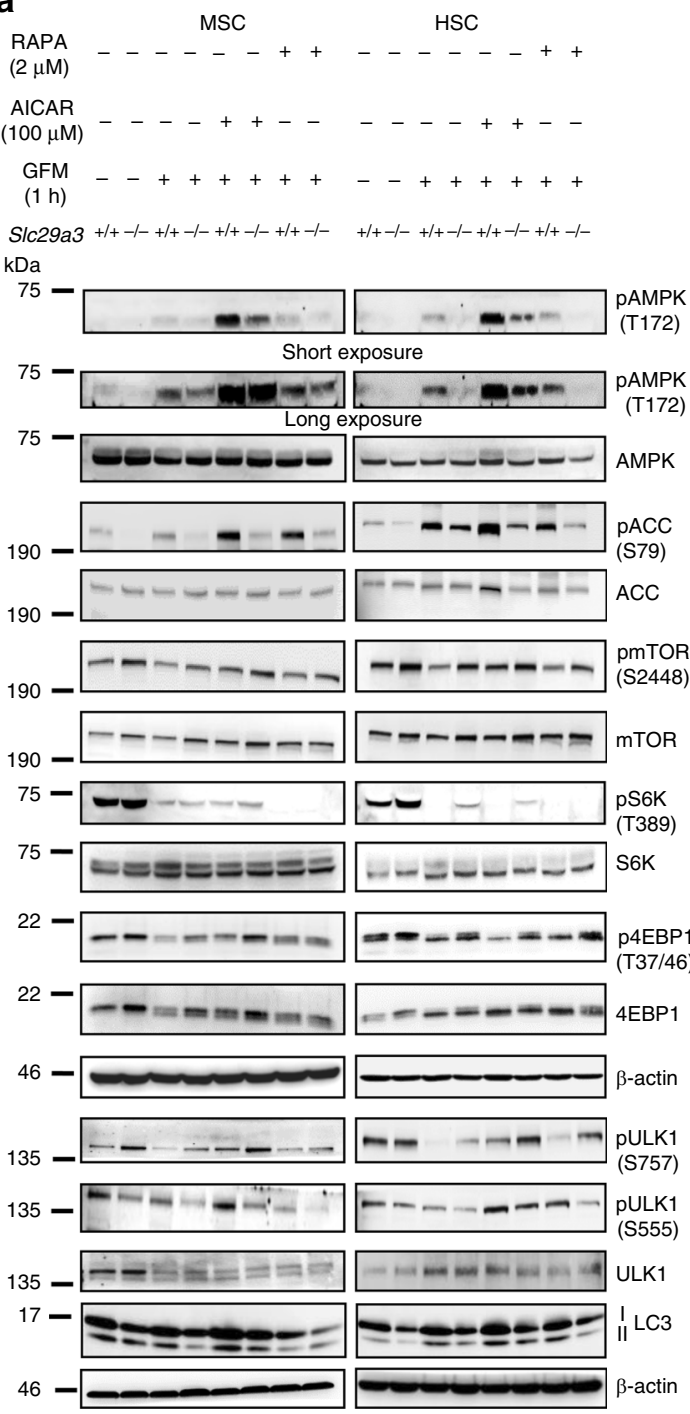

b
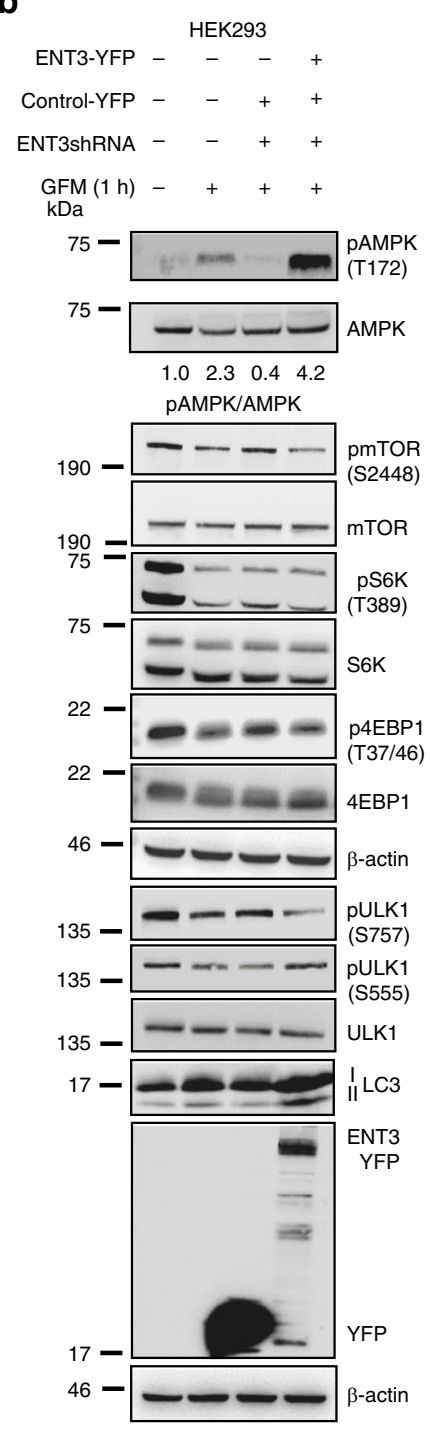

C

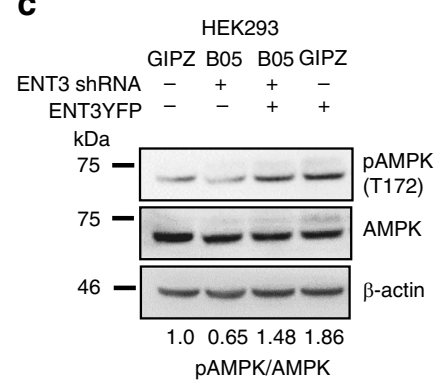

d
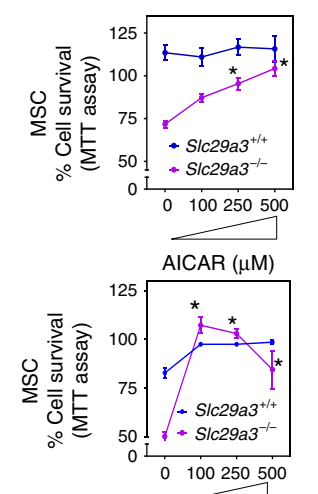

$1.5 \operatorname{AICAR}(\mu \mathrm{M})$
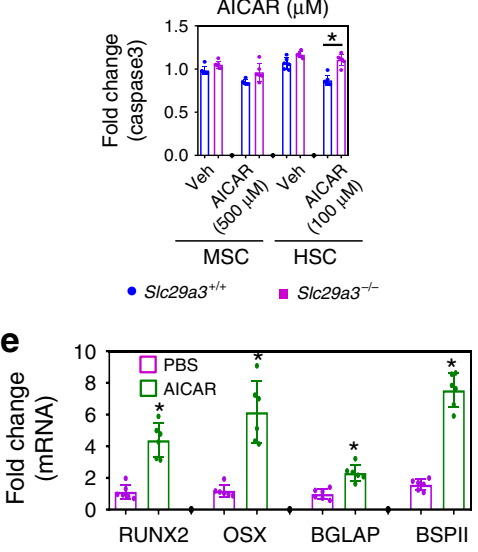
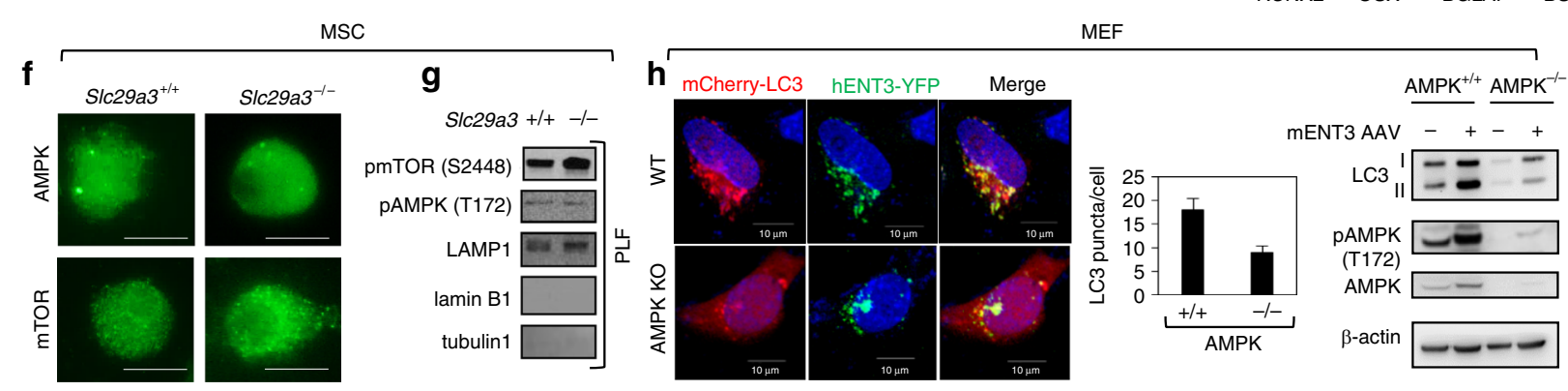

addition to the gain in CFU-F formation in the SCT group (Fig. 9h, i and Supplementary Fig. 11a). Furthermore, the MSC differentiation abilities in SCT mice were substantially rescued as assessed by the levels of lineage-specific markers and transcription factors (Fig. 9j).

Consistent with the gross observations in medullary regions showing improved hematopoiesis in the AICAR-treated and SCT groups (Fig. 9b), blood analysis revealed a trend of an increase in RBCs, the hemoglobin content and the platelet count with a decrease in the granulocytic and agranulocytic WBC counts (Fig. 9k). Compared with the AICAR-treated group, the SCT group showed a superior rescue effect for many parameters

(Fig. 9k). When bone marrow erythroid subpopulations were further characterized, we observed a reversal of the changes in the proportion of cells at various erythroblast maturation stages in the SCT group compared with that in $S l c 29 a 3^{-1-}$ mice at 12 weeks of age (Fig. 91 and Supplementary Fig. 11b). The reduced proportion of immature stages (proerythroblasts (ProE), basophilic erythroblasts (EryA), late basophilic erythroblasts (EryB)) observed in $S l c 29 a 3^{-1-}$ mice at 12 weeks of age was increased in the SCT group to levels beyond that seen in the WT group, suggesting the expansion of erythroid precursors in the SCT group (Fig. 91). This shift towards an immature population resulted in a percent decrease in the mature 
Fig. 5 ENT3 activates the AMPK signaling pathway. Representative immunoblots of proteins involved in the AMPK-mTOR-ULK axis in MSCs and HSCs derived from 12-week-old mice under basal, glucose-starved (GFM), and AICAR- or rapamycin (RAPA)-treated conditions (a). Immunoblot analysis of the AMPK-mTOR-ULK axis in HEK293-expressing control-shRNA, ENT3-shRNA, pE-YFP, or pEYFP-ENT3 subjected to GFM (b). Immunoblotting and quantification of the ENT3 shRNA inhibition of AMPK phosphorylation and reversal by ENT3YFP in HEK293 (c). Effect of AICAR on GFM-induced MSC and HSC survival and death as assayed by MTT assay and active caspase 3 measurement, respectively $(n=6$, mean \pm SEM). The ' 0 " concentration compares

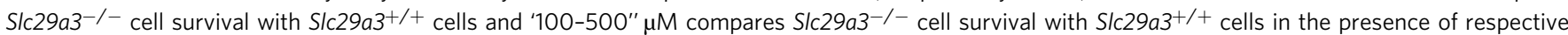
$\operatorname{AICAR}$ concentrations $(n=4$, mean \pm SEM). HSCs and MSCs were derived from 12-week-old mice (d). Effect of AICAR (500 $\mu M)$ on osteogenic medium ( 3 days)-induced transcription factors (TF) and marker genes expression ( $n=6$, mean \pm SEM) in S/c29a3 ${ }^{-/-}$MSCs. HSCs and MSCs were derived from 12-week-old mice (e). Immunostaining analysis of AMPK (green) and mTOR (green) in Slc29a3-/- and Slc29a3 ${ }^{+/+}$MSCs (derived from 12-week-old mice). Original magnification, $\times 60$; Scale bar: $10 \mu \mathrm{m}$ (f). Immunoblot analysis of pmTOR, pAMPK, LAMP1 (lysosomal marker), lamin B1 (nuclear marker), and tubulin 1 (cytoplasmic marker) in lysates prepared from purified lysosomal fraction (PLF) isolated from Slc29a3 ${ }^{-/-}$and Slc29a3 ${ }^{+/+}$MSCs (derived from 12-week-old mice) (g). WT and AMPK KO ( $\left.\alpha 1 / \alpha 2^{-/-}\right)$MEFs were transfected with mCherry-LC3 and pEYFP-hENT3 plasmids and LC3 and YFP fluorescence were visualized and quantified $(n=3$, mean \pm SEM). Scale bar: $10 \mu \mathrm{m}$. Immunoblots of AMPK, pAMPK, and LC3 forms in WT and AMPK KO MEFs transduced with AAV harboring GFP (-) or mENT3 (+) (right) (h). GFM, glucose-free medium. Statistical analyses were performed using ANOVA with Tukey's multiple comparisons post-test and two-tailed Student's $t$-test. ${ }^{\star} P<0.05$. Source data are provided as a Source Data file

orthochromatic/reticulocyte erythroblast (EryC) in the SCT group (Fig. 91). However, the findings regarding the erythroid frequencies with increased bone marrow cellularity (and \% HSCs in SCT group) (Fig. 9m) suggested that the absolute numbers of HSCs and immature cell types (ProE to EryB) are dramatically increased while the number of mature erythroblasts (EryC) is still maintained in the SCT group (relative to Slc29a3 ${ }^{-1-}$ mice at 12 weeks of age). The increase in the percentage of HSCs further suggested that the bone marrow regeneration might have resulted from the continuous proliferation and self-renewal of the primitive HSCs in the SCT group (Fig. 9m). To examine this directly, we assessed the changes in the HSC number and expression of surface markers during in vitro proliferation with serial passage. Abnormal proliferation with the loss of markers in Slc29a3 ${ }^{-1}$ HSCs was normalized in the SCT group, suggesting a recovery of self-renewal (Fig. 9n, o and Supplementary Fig. 11c). Similarly, the abnormal increase in myeloid subsets (CFU-G, CFU-M, and CFU-GM) with the loss of BFU-E was normalized in the SCT group with corresponding changes in erythroid and myeloid transcription factors and markers (Fig. 9p). In addition to SCT, AICAR treatment moderately increased erythroid (ProE, EryA, and EryB) frequencies (Fig. 91 and Supplementary Fig. 11b) and bone marrow cellularity (Fig. 9m), however, sample limitation from AICAR-treated surviving mice at the end of the treatment period precluded us from conducting many detailed studies.

To study the effect of AICAR on freshly isolated stem cells, we treated another batch of Slc29a3 $3^{-1-}$ mice with saline or AICAR (daily; $50 \mathrm{mg} / \mathrm{kg}$; intraperitoneal) for a shorter period (6 weeks, starting at 10 weeks) and examined for changes in HSC and MSC pools. Consistent with the results on continuous administration of AICAR on Slc29a3 $3^{-1-}$ mice survival (for 28 weeks), even the AICAR-treated younger Slc29a3 ${ }^{-1-}$ mice (at 16 weeks) showed improvement in the number of HSCs and MSCs (Supplementary Fig. 12a-d). Further, lysates prepared from the bone marrow and spleens isolated from the AICAR-treated mice at 16 weeks showed increased LC3-II levels (Supplementary Fig. 12e). These results provided further support that the AICAR induced increases in HSC and MSC abilities contribute to the partially reduced ENT3 disease pathologies and improved survival of Slc29a3 ${ }^{-1-}$ mice.

\section{Discussion}

It is increasingly evident that mutations in the ENT3 gene are solely responsible for a growing spectrum of human genetic disorders ${ }^{5-16}$. However, due in part to the complexity and heterogeneity of ENT3-related dysfunctions, the origin of dysfunction and molecular mechanism(s) by which ENT3 regulates cellular and tissue homeostasis have remained obscure. Here, we first established a role for ENT3 in the self-renewal and differentiation capabilities of multipotent MSCs and HSCs. Second, we identified that ENT3 mobilizes lysosomal adenosine pool to regulate AMPK-mTOR-ULK signaling, the disruption of which leads to widespread autophagic insufficiency. Third, we found that the impairment of differentiation capabilities of HSCs and MSCs in ENT3-null mice was associated with stem cell exhaustion and breaches of mesodermal tissue integrity. Finally, stem cell transplantation demonstrated the dramatic rescue of tissue pathologies and survivability in ENT3-deleted mice, whereas the pharmacological activation of AMPK by AICAR produced a partial recovery. Altogether, these discoveries identified the involvement of an intracellular nucleoside transporter in adult stem cell homeostasis. Additionally, the data provide a mechanistic explanation for the complex and heterogeneous tissue pathologies observed in ENT3-related disorders (Fig. 10). Moreover, the findings support an intriguing possibility that ENT3 senses the lysosomal adenosine levels and determines the activation levels of the central energy sensor (AMPK), which, in turn, relays the information to core autophagic machinery to ensure normal tissue and organismal development (Fig. 10).

Our study provides the evidence for the impairment of selfrenewal and differentiation functions in ENT3-deficient MSCs and HSCs through perturbation of the transport of adenosine across lysosomal membranes. As Slc29a3 $1-1-$ mice largely phenocopy the abnormalities reported in autophagy-deficient mod$\mathrm{els}^{31,37,50,51}$ and autophagic induction via AMPK activation partially ameliorates dysfunctions, our findings support that the loss of autophagic signaling as a probable cause of stem cell dysfunction in ENT3 KO mice. Overall, the evidence support that the autophagy inducing effects of AMPK pathway to contribute to the survival of ENT3-deficient cells although additional contributions from the autophagic-independent effects of this multifunctional kinase cannot be completely negated. The preponderance of dysfunction in mesoderm-derived tissues demonstrates that the defects originated at the lower hierarchical MSCs and HSCs and not the higher hierarchical cells, a phenomenon that would be expected to produce abnormalities in all three germ layers. This dysfunction also suggests that MSCs and HSCs exhibit a higher dependency on ENT3-regulated autophagic needs to sustain mesodermal tissue integrity. Our study also uncovered that ENT3 mobilizes the intracellular pool of adenosine from the lysosomes to increase AMPK phosphorylation, a process that directly activates autophagy. The constitutively active mTOR activity downstream to AMPK loss also indicates a higher energy state in Slc29a3-1- cells that supports cell survival mechanisms but compromises the ability to adapt to higher 
a

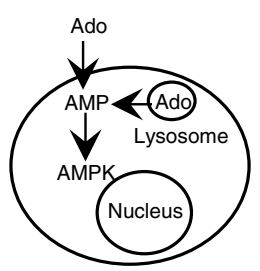

C

- Lysosomal acid phosphatase

- Lysosomal Adenosine

- Cytosolic Adenosine

- Cytosolic AMP
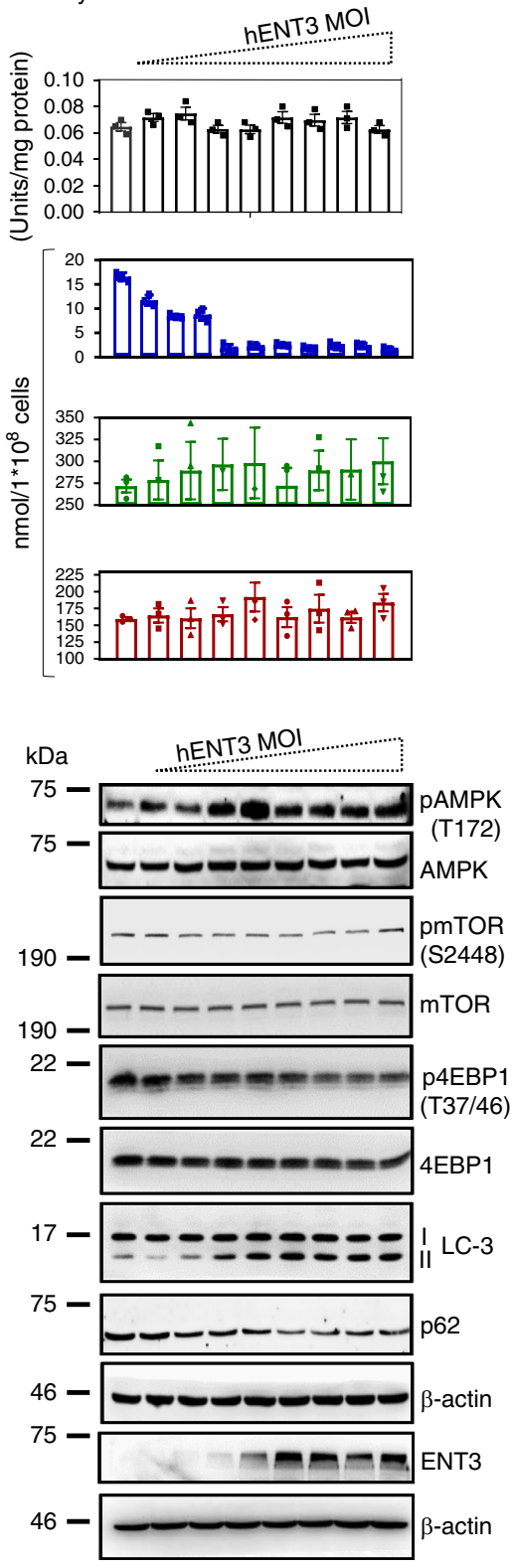

b

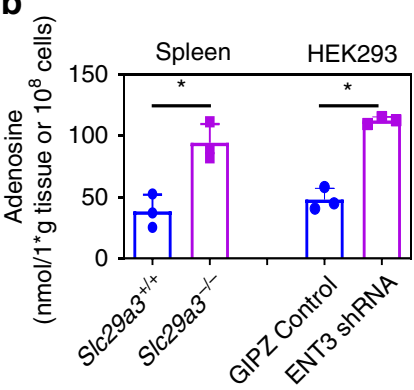

d
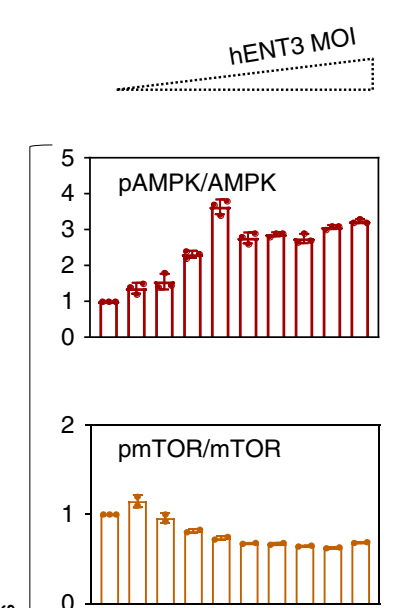

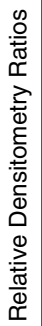
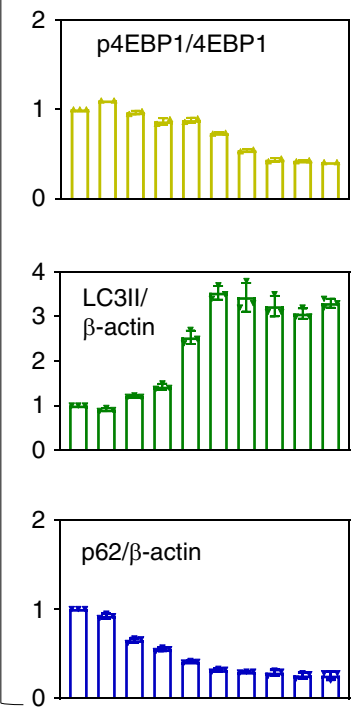

e

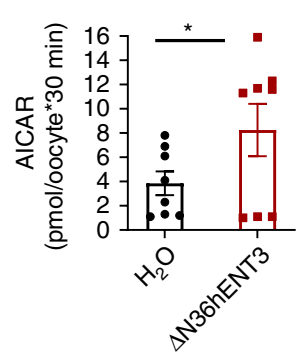

f

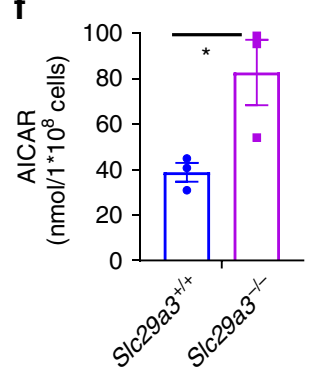

$\mathbf{g}_{5^{\prime} \text {-lodotubercidin } \ldots \ldots+}$

ENT3YFP --++
peYFP ++--
GFM $(1 \mathrm{~h})-+++$

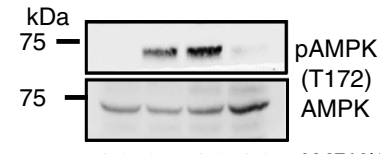

$1.02 .5 \quad 4.3 \quad 1.8$ pAMPK/AMPK
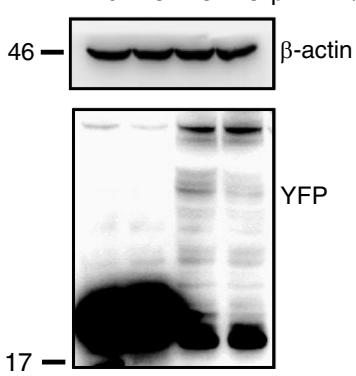

h

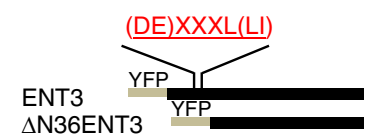

i

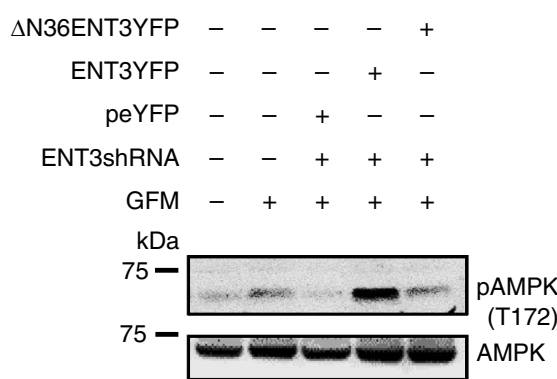

$\begin{array}{llllll}\text { pAMPK/AMPK } & 1.0 & 1.73 & 0.75 & 3.6 & 1.83\end{array}$

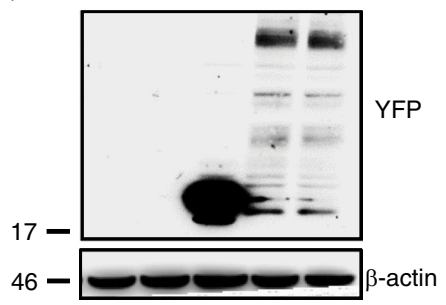

energy demands via autophagy. Thus, the present findings indicate ENT3 intricately regulates autophagy by involving both AMPK and mTOR signaling pathways.

In summary, by evaluating the mutational consequences of hENT3 disorders in ENT3-deficient mice, we investigated hallmarks of hENT3 pathophysiologies and evaluated therapeutic interventions. Forced activation of AMPK by AICAR partially ameliorates the severity of ENT3-related dysfunction, whereas stem cell transplantation appears even more promising with dramatic survival benefits in mice. Altogether, these findings have important implications to understand ENT3 pathophysiology and to treat humans with ENT3 disorders. 
Fig. 6 ENT3 lysosomal adenosine transport regulates AMPK and autophagy signaling. Schematic representation of the activation of AMPK by extracellular and lysosomal pools of adenosine; Ado, adenosine; AMP, adenosine monophosphate (a). Mass spectrometry analysis of adenosine levels in lysosomes derived from mouse spleen lysates and HEK293 cells expressing GIPZ-shRNA (control) or ENT3 shRNA $(n=3$, mean \pm SEM) (b). HEK293 cells transduced with increasing $\mathrm{MOI}$ of hENT3 retroviruses were analyzed for lysosomal adenosine and cytosolic adenosine and AMP, and lysosomal acid phosphatase activity $(n=3$, mean \pm SEM) (c; above). Whole-cell lysates prepared from cells infected with the corresponding MOls of hENT3 viruses were probed with the indicated antibodies. $\beta$-Actin served as the loading control (c; below). Quantification results from densitometric analysis of the immunoblots $(n=3$, mean \pm SEM) $(\mathbf{d})$. Uptake of AICAR $(20 \mu \mathrm{m})$ into Xenopus oocytes at $25^{\circ} \mathrm{C}$ after $22 \mathrm{~h}$ of injection of N-terminal deleted ENT3 transcripts $(\Delta \mathrm{N} 36 \mathrm{ENT3}$; to remove the lysosomal-targeting signal (shown in (h)) and enabling cell surface localization) was measured in transport buffer with pH 5.5. AICAR concentrations measured by mass spectrometry ( $n=8$ oocytes, mean \pm SEM) (e). Mass spectrometry analysis of AICAR levels in lysosomes derived from MSCs derived from 12-week-old mice after the treatment of MSCs with AICAR (20 $\mu \mathrm{m})$ for $4 \mathrm{~h}(n=3$, mean \pm SEM) (f). HEK293 cells cultured in GFM were transfected with pE-YFP or pE-ENT3-YFP and treated with iodotubericidin $(50 \mathrm{nM})$ for $4 \mathrm{~h}$ and lysates examined for indicated proteins. lodotubericidin abrogates glucose starvation and ENT3-induced pAMPK activation. GFM, glucose-free medium (g). Schematic representation of WT ENT3 (ENT3) and localization-deficient ENT3 ( $\triangle$ N36ENT3) mutants fused with YFP. A lysosomal-targeting motif in the N-terminus of ENT3 (red) and YFP tag (yellow) at the N-terminus are shown (h). HEK293 cells transduced with WT ENT3 but not N-terminal deleted ENT3 elicit glucose starvation-induced pAMPK activation (i). Statistical analyses were performed using two-tailed Student's $t$-test. ${ }^{\star} P<0.05$. Source data are provided as a Source Data file

\section{Methods}

Cells, plasmids, reagents, and antibodies. Retroviral (ATCC, CRL 9078) and lentiviral packaging (ATCC, CRL 11268) cell lines and HEK 293 cells (ATCC, CRL 1573) were purchased from American Type Culture Collection (ATCC, Manassas, VA, USA). AMPK $\alpha 1 / \alpha 2$ double-knockout MEFs were a kind gift from Dr. B. Viollet (Institut Cochin INSERM U1016, CNRS UMR 8104, Université Paris Descartes, Department of Endocrinology, Metabolism and Cancer, Paris, France. Each cell line was tested negative for mycoplasma contamination (MycoAlertTM Mycoplasma Detection kit, Lonza).

pEYFP-hENT3, pOX- $\Delta$ N36hENT3, LNCX2- $\Delta$ N36hENT3, and mRFP-EGFPLC3 plasmid constructs were described previously ${ }^{18,20}$. Human SLC29A1 and hSLC29A2 were cloned into pEYFP-C1 (Clontech, Mountain View, CA) and were designated as pEYFP-hENT1 and pEYFP-hENT2, respectively. The pcDNA3.1mCherry-hLC3B plasmid (40827) was obtained from Addgene ${ }^{52}$. The GIPZ lentiviral vector harboring shRNA sequences targeting SLC29A3 (RHS4531EG55315) and a non-targeting control-shRNA were obtained from Dharmacon, Chicago, IL. The pAAV-mSlc29a3 (Cat. AAV0925011), pAAV-CMV-GFP-control (Cat. AAV1000), pLenti-GIII-CMV-ATG7 (Cat. LV082738) and pLenti-GIIISlc29a3-Puro (Cat. LV451098) plasmid constructs were obtained from Applied Biological Materials Inc. (Richmond, BC, Canada).

All chemicals were procured from Sigma (St. Louis, MO) unless otherwise indicated. Rapamycin was purchased from Enzo Life Sciences, and Torin1 was purchased from Sellekchem (Houston, TX). The BCA protein assay kit was purchased from Thermo Scientific, the lentiviral packaging kit was purchased from Dharmacon (Chicago, IL, USA) and the adeno-associated viral packaging kit was purchased from Applied Biological Materials Inc. (Richmond, BC, Canada). Fetal bovine serum and horse serum were from HyClone Laboratories (Logan, UT). Dialyzed fetal bovine serum was from Sigma (St. Louis, MO). Fluorescent Antifade mounting reagent and penicillin-streptomycin were obtained from Molecular Probes (Eugene, OR). High-glucose DMEM and no-glucose DMEM cell culture media were obtained from Thermo Scientific (Waltham, MA).

Goat polyclonal antibodies against carboxyl (C20; sc-48147; 1:1000 dilution) or amino (N18; sc-48149; 1:1000 dilution) terminus of hENT3 were obtained from Santa Cruz Biotechnology (Santa Cruz, CA), and the rabbit polyclonal antibody generated against the third intracellular loop of hENT3 was described earlier ${ }^{20}$. The rabbit polyclonal anti-hENT3 antibody (PA5-38039; 1:1000 dilution) was purchased from Thermo Scientific (Waltham, MA). Rabbit monoclonal antibodies against LC3B (\#3868; 1:1000 dilution), pmTOR (\#5536; 1:1000 dilution), mTOR (\#2983; 1:1000 dilution), p4EBP1 (\#2855; 1:1000 dilution), 4EBP1 (\#9644; 1:1000 dilution), PS6K (\#9234; 1:1000 dilution), S6K (\#2708; 1:1000 dilution), pULK1S555 (\#5869; 1:500 dilution), pULK1-S757 (\#14202; 1:500 dilution), ULK1 (\#6439; 1:200 dilution), pACC (\#11818; 1:1000 dilution), ATG7 (\#8558; 1:1000 dilution), and Beclin1 (\#3495; 1:1000 dilution) and rabbit polyclonal antibodies against pAMPK (\#2531; 1:200 dilution), AMPK (\#2532; 1:1000 dilution), ACC (\#3662; 1:1000 dilution), ATG5 (\#2630; 1:1000 dilution), lamin B1 (\#12586; 1:1000 dilution), tubulin (\#2125; 1:1000 dilution) and LAMP1 (\#D2D11; 1:200 dilution) were obtained from Cell Signaling Technology (Beverly, MA). Antibody conjugates for FLT3 (APC-anti-CD135, clone A2F10.1; 1:50 dilution), IL7Ra (V450-antiCD127, clone SB/199; 1:50 dilution), Sca1 (PE-Cy7-anti-Ly-6A/E, clone E13-161.7; 1:50 dilution), and CD34 (FITC-anti-CD34, clone RAM34; 1:50 dilution) were obtained from BD Biosciences (San Jose, CA), while c-kit (PE-anti-CD117, clone REA791; 1:50 dilution) was obtained from Miltenyi Biotech (Bergisch Galadbach, Germany). Additionally, BUV395-anti-Scal (clone D7; 1:50 dilution) and PE-Cy7anti-c-kit (Clone 2B8; 1:50 dilution) antibodies were purchased from BD Biosciences (San Jose, CA) and PE-anti-FLT3 (clone A2F10; 1:50 dilution) was purchased from eBioscience. Rabbit polyclonal antibodies against EEA-1 (1:1000 dilution) and calreticulin (1:1000 dilution) and hsp70 (1:1000 dilution) mouse monoclonal antibodies were obtained from Affinity Bioreagents (Golden, CO). The mouse monoclonal Anti-SQSTM1 (p62) antibody (\#610833; 1:500 dilution) was purchased from BD Biosciences, anti- $\beta$-actin antibody (\#A5316; 1:1000 dilution) was purchased from Sigma (St. Louis, MO) and anti-GFP antibody (sc-9996; 1:1000 dilution) was purchased from Santa Cruz Biotechnology (Santa Cruz, CA) Anti-F4/80 antibody (ab6640; $1: 1000$ dilution) and anti- $\beta$-COP antibody (ab2899; 1:200 dilution) from were obtained from Abcam. Horseradish peroxidaseconjugated anti-rabbit IgG, anti-mouse IgG and anti-goat IgG (1:2000 dilutions) were purchased from Bethyl Laboratories (Montgomery, TX). Alexa 488- and 594conjugated anti-rabbit, anti-mouse, and anti-goat secondary antibodies (1:2000 dilutions) were obtained from Invitrogen, Thermo Scientific (Waltham, MA).

Isolation and maintenance of MSCs and HSCs. The mice were euthanized with $\mathrm{CO}_{2}$ asphyxiation, and the bone marrow from the femur and tibiae was flushed with stem cell medium [(minimum essential medium with no nucleosides (a-MEM, ThermoFisher Scientific) containing 1\% L-glutamine, 10\% fetal bovine serum, $100 \mathrm{U} / \mathrm{ml}$ of penicillin, and $100 \mathrm{mg} / \mathrm{ml}$ of streptomycin)] and filtered through a $40-\mu \mathrm{m}$ pore size cell strainer (BD Biosciences, Bedford, MA). For HSC isolation, freshly harvested bone marrow cells were c-kit-enriched using c-kitconjugated magnetic beads (Miltenyi, Bergisch Gladbach, Germany), and subsequently costained with antibodies against lineage markers (Lineage Cell Detection Cocktail, Miltenyi), FLT3 (APC-anti-CD135), IL7Ra (V450-anti-CD127), c-kit (PE-anti-CD117), Sca1 (PE-Cy7-anti-Ly-6A/E), and CD34 (FITC-anti-CD34). In other instances, lineage-positive $\left(\mathrm{Lin}^{+}\right)$cells were depleted by magnetic-activated cell sorting (MACS) using the MagCellect mouse hematopoietic cell lineage depletion kit (R\&D Systems, Minneapolis, MN). The Lin ${ }^{-}$cells from MACS were again gated in FACS for the negative selection of Lin + populations using the APC mouse lineage antibody cocktail (BD Biosciences, San Jose, CA). Next, the Lin ${ }^{-}$cells were gated using BUV395-anti-Sca1, PE-Cy7-anti-c-kit, PE-anti-FLT3 and FITC-anti-CD34 antibodies and were sorted to obtain

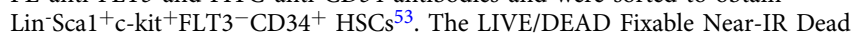
Cell Stain Kit (ThermoFisher Scientific, Waltham, MA) was used to exclude dead cells. Cell staining was performed on ice and analyzed using a BD LSRFortessa equipped with five lasers $(355,405,488,561$, and $633 \mathrm{~nm})$. Color compensation was performed using BD FACSDIVA Software (BD Biosciences, Bedford, MA) and FACS data analysis was performed using FlowJo (Tree Star, Ashland, OR).

Long-term HSC (LT HSC), short-term HSC (ST HSC), and multipotent progenitor (MPP) cell types were quantified following gating schemes described previously ${ }^{54}$. In short, cells exhibiting a $\mathrm{Lin}^{-} \mathrm{IL}_{\mathrm{RRa}}{ }^{-} \mathrm{Scal}^{+} \mathrm{c}-\mathrm{kit}^{+}$phenotype were resolved into LT HSC, ST HSC, or MPP cell types based on differential expression of CD34 and FLT3 ${ }^{55}$. Among $\mathrm{Lin}^{-} \mathrm{IL}_{\mathrm{R}} \mathrm{Ra}^{-} \mathrm{Sca}^{+} \mathrm{c}$-kit ${ }^{+}$cells, LT

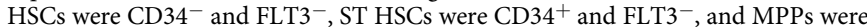
$\mathrm{CD}_{3} 4^{+}$and $\mathrm{FLT}_{3}{ }^{+}$. HSCs were maintained in HSC medium [serum-free stem cell expansion medium (StemSpan, Stem Cell Technologies) supplemented with Stem Cell Factor (SCF) $(100 \mathrm{ng} / \mathrm{ml})(\mathrm{R} \& \mathrm{D}$ systems)] for downstream applications.

For MSCs, the Lin ${ }^{-}$cells were allowed to adhere for 3 days in stem cell medium. The non-adherent hematopoietic cells were discarded, and the adherent populations were trypsinized and expanded for 2 weeks. BUV395-anti-Sca1, PerCP-Cy5.5-anti-CD44, PE-CF594-anti-CD49e, APC-anti-CD90 and BV510anti-CD105 antibodies (BD Biosciences) were used to obtain $\mathrm{Lin}^{-} \mathrm{Sca}{ }^{+}{ }^{+} \mathrm{CD} 44^{+}$ $\mathrm{CD} 49 \mathrm{e}^{+} \mathrm{CD} 90^{+} \mathrm{CD}_{105^{+}} \mathrm{MSCs}^{53,56}$. MSCs were maintained in stem cell medium (as detailed above) for all experiments.

Gating strategies for flow cytometry analysis of HSC and MSC in various experiments are indicated in Supplementary Fig. 13.

Differentiation of MSCs and HSCs. Adipogenic or osteoblastogenic differentiation of MSCs was induced using established methods ${ }^{57}$. Mineralization was performed by Alizarin red-S staining 57 . Chondrocyte differentiation was induced using a commercial StemPro chondrogenesis differentiation kit (Thermo Scientific, Waltham, MA). Alcian blue staining was used to detect collagen production with 
a
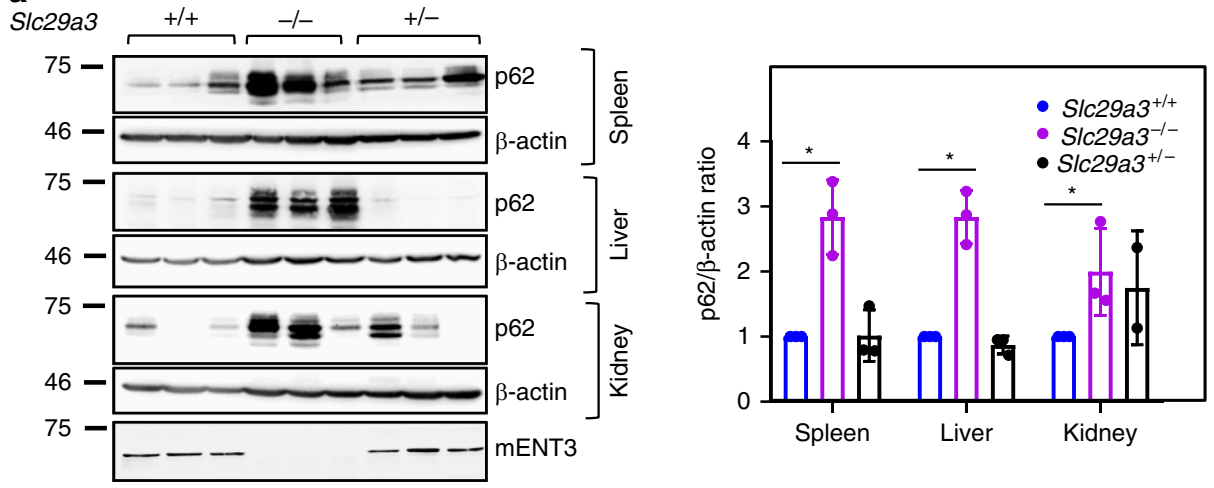

b

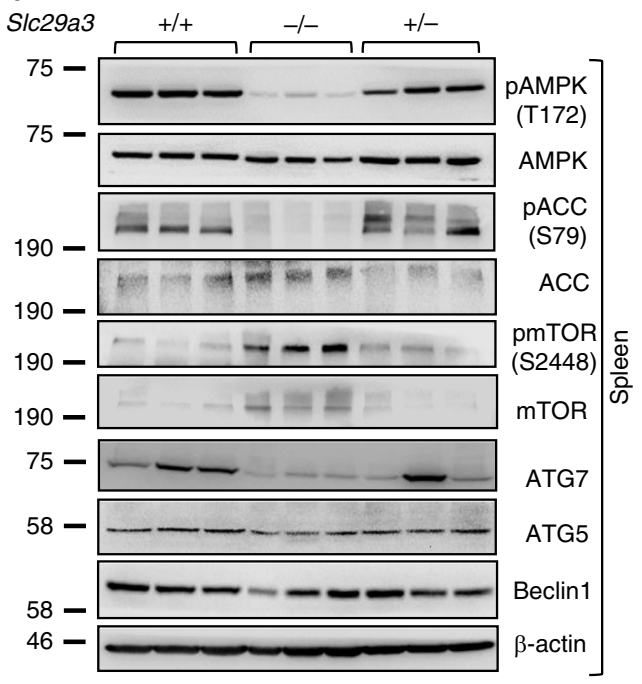

C

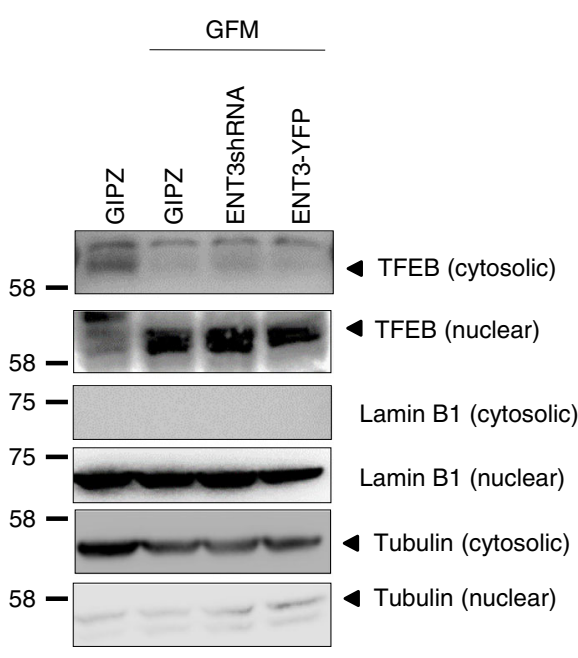

d

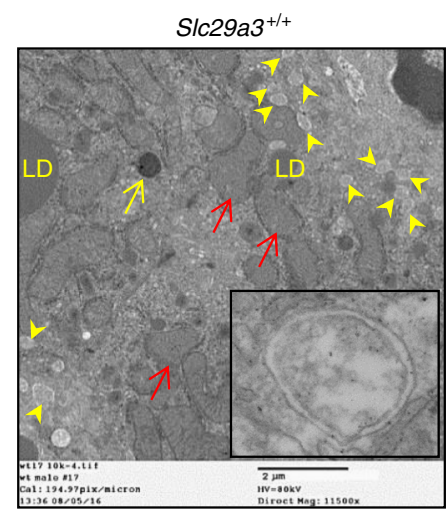

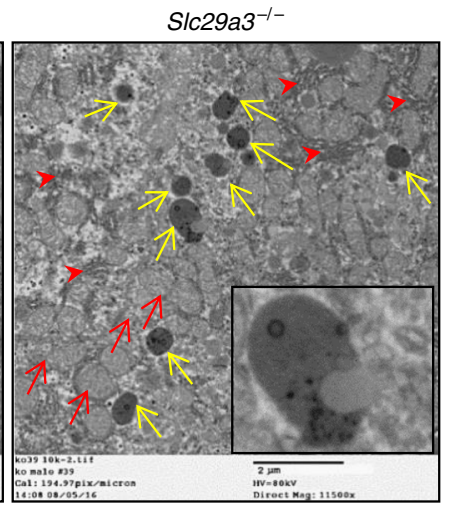

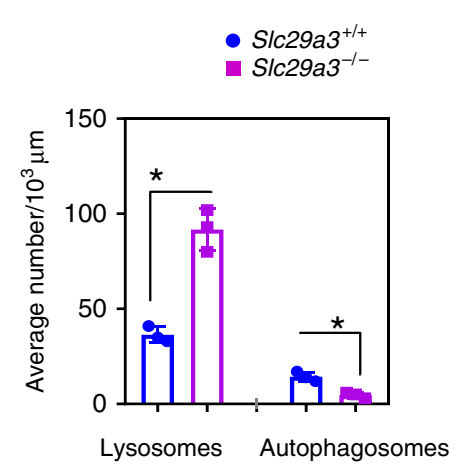

Fig. 7 Autophagy perturbation in S/c29a3-/- adult mouse tissues. Immunoblotting analysis (left) and quantification (right) of lysates prepared from the Slc29a3-/- spleen, liver, and kidney showing the accumulation of p62 compared with that in S/c29a3+/+ tissues (a). Immunoblotting analysis of the AMPK-mTOR-ULK axis and core autophagy genes in S/c29a3 $+/+$, Slc29a3 ${ }^{-/-}$and Slc29a3 ${ }^{+/-}$splenic lysates (b). HEK293 cells were transfected with control or hENT3YFP plasmids, and the cytosolic and nuclear fractions were isolated and examined for alterations in the TFEB levels using immunoblotting analysis. No significant changes in the TFEB levels were identified in the presence or absence of hENT3. Lamin B1 was used as a nuclear marker, and tubulin was used as a cytosolic marker. $\beta$-Actin served as the loading control (c). TEM analysis (left) and quantification (right; $(n=3$, mean \pm SEM)) of lysosomes (yellow arrow) and autophagosomes (yellow arrowhead) in S/c29a3 ${ }^{+/+}$and S/c29a3-/- mouse livers. Insets show representative lysosome and

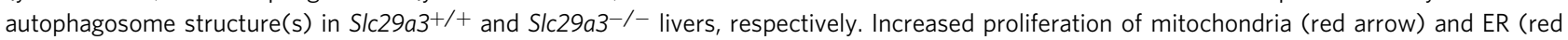
arrowhead) in S/c29a3-/- livers. LD, lipid droplets; Scale bars: $2 \mu \mathrm{m}$ (d). Statistical analyses were performed using two-tailed Student's $t$-test. ${ }^{\star} P<0.05$. Source data are provided as a Source Data file

chondrocyte differentiation ${ }^{58}$. Myocyte differentiation was induced by treating MSCs with $2 \%$ horse serum ${ }^{59}$. Cell nuclei were counterstained with $1 \mu \mathrm{g} / \mathrm{ml}$ of Hoechst 33258 (Sigma-Aldrich). A detailed description of these methods is available in the Supplementary Methods.

HSCs were cultured in complete methylcellulose media, and their differentiation potential was assessed by colony-forming cell assays (MethoCult GF
M3434 (Stem Cell Technologies)). Burst-forming units-erythroid (BFU-E), colonyforming units-macrophage (CFU-M), colony-forming units-granulocyte (CFU-G), and colony-forming unit-granulocyte/ macrophage (CFU-GM) were characterized and scored according to their morphology ${ }^{60}$. Osteoclast differentiation of HSCs was induced by $30 \mathrm{ng} / \mathrm{ml}$ of M-CSF (R\&D Systems) and $50 \mathrm{ng} / \mathrm{ml}$ of RANKL (R\&D Systems) for 14 days $^{53}$. On day 14, the osteoclasts were fixed and stained with 


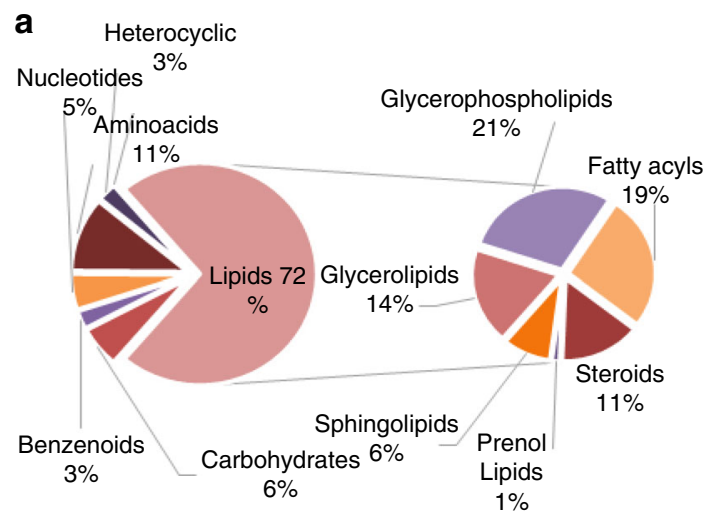

C

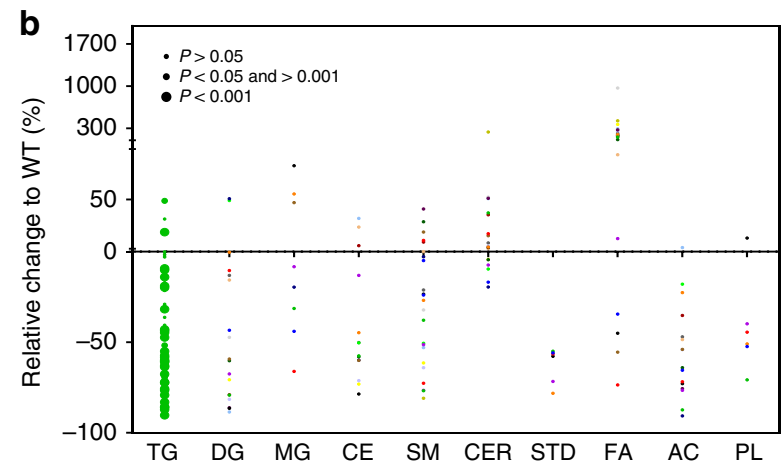

d

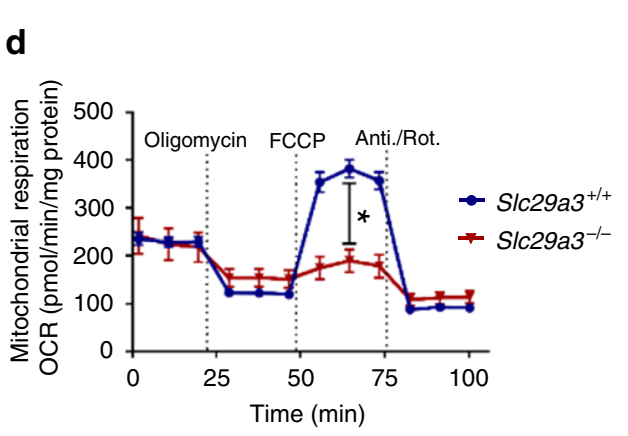

e
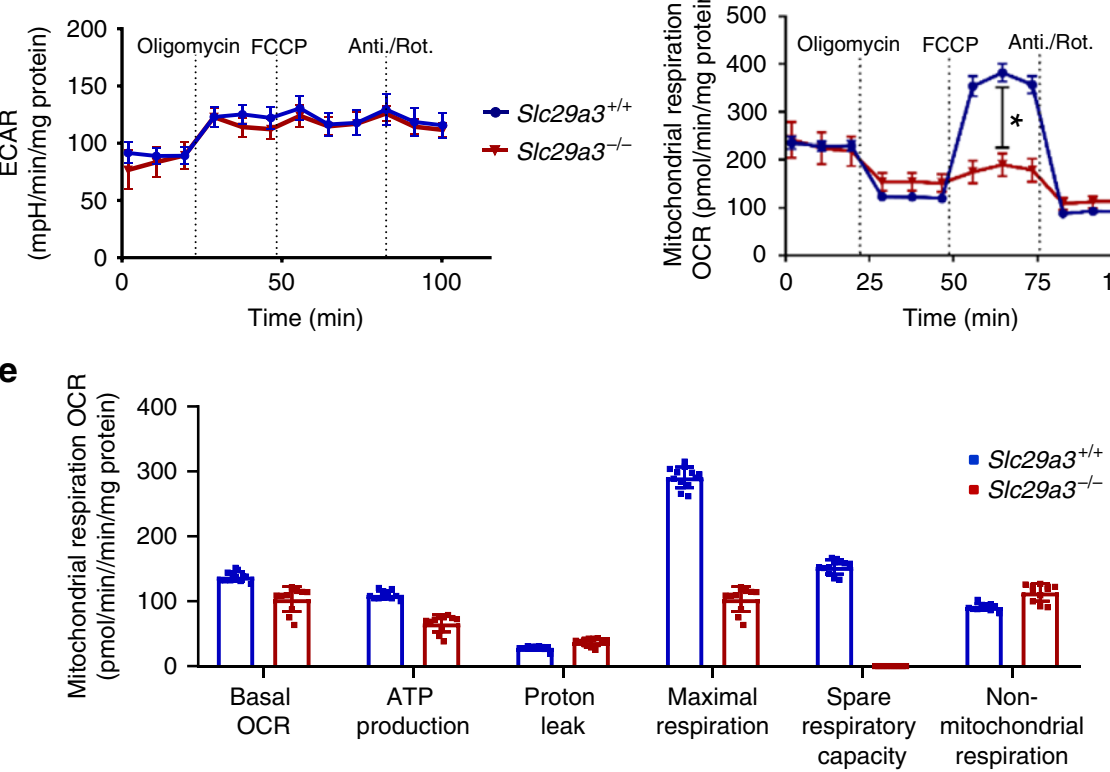

f
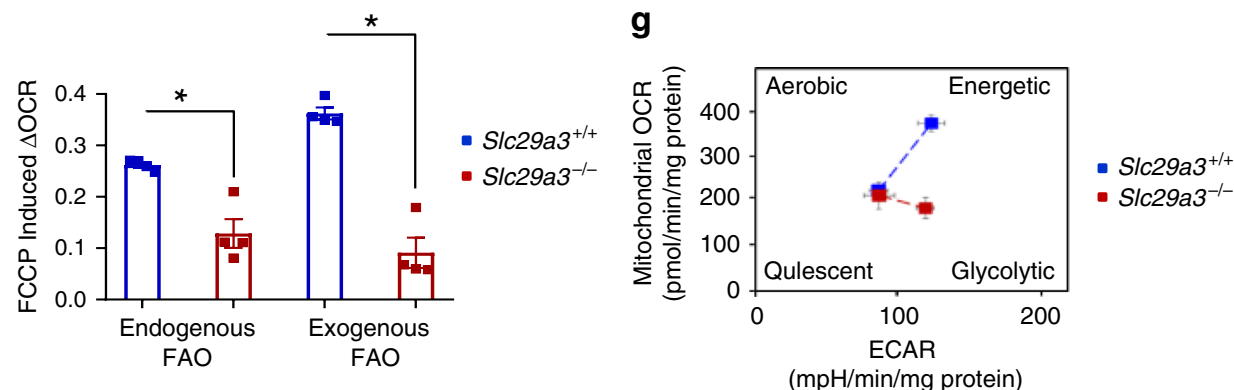

Fig. 8 Disrupted lipid homeostasis and altered fuel source selection in ENT3 deficiency. Pie-of-pie chart representation of total metabolites and those within the lipid superclass in the S/c29a3-/- liver (a). Bubble chart representation of quantified lipids by targeted lipidomics (shown in Supplementary Fig. 9). Bubble, individual lipid; bubble size, significance (inset); $n=12$ (b). Real-time measurements of the extracellular acidification rate (ECAR) (c) and oxygen consumption rate (OCR) (d) in S/c29a3-/- MSCs $(n=11$, mean \pm SEM). In addition to the basal levels, the fraction of OCR and ECAR contributing to ATP-linked OCR/ECAR measured by sequential injection of oligomycin, FCCP and antimycin/rotenone (Anti/Rot) are presented (c, d). Mitochondrial substrate utilization parameters in S/c29a3-/- MSCs derived from $\mathbf{c}$ and $\mathbf{d}(\mathbf{e})$. Quantification of the oxidation of endogenous and exogenous palmitic acid

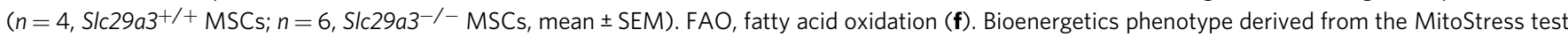
(g). Statistical analyses were performed using two-tailed Student's $t$-test. ${ }^{\star} P<0.05$. Source data are provided as a Source Data file

tartrate-resistant acid phosphatase (TRAP) using a leukocyte acid phosphatase kit (Sigma-Aldrich, St. Louis, MO) or were subjected to visualization of actin ring formation using tetramethylrhodamine-phalloidin (Life Technologies, Carlsbad, CA) ${ }^{53}$.

Retroviral production and transduction. A retroviral packaging cell line (ATCC, CRL 9078) was maintained in Dulbecco's modified minimum essential medium
(DMEM) supplemented with $5 \%$ fetal bovine serum. These cells were transfected with retroviral plasmid constructs harboring SLC29A3 or $\triangle \mathrm{N} 36 S L C 29 A 3^{18,20}$ for viral production. Cells were incubated with a complex containing $8 \mu \mathrm{g}$ of each of the plasmids, $24 \mu \mathrm{l}$ of FuGENE 6 reagent (Roche Applied Science), and $800 \mu \mathrm{l}$ of Opti-MEM serum-free medium (Invitrogen). After $48 \mathrm{~h}$ of transfection, viruses in the supernatant were harvested, filtered, and target cells were transduced with recombinant viruses at $4 \mu \mathrm{g} / \mathrm{ml}$ polybrene to virus concentration. 
a

- - Slc29a3 ${ }^{+/ 4}(\mathrm{WT})(n=10)$

- - . Slc29a3 ${ }^{-1-}+$ saline $(n=10)$

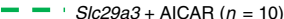

- - - SIc29a3 + SCT $(n=16)$

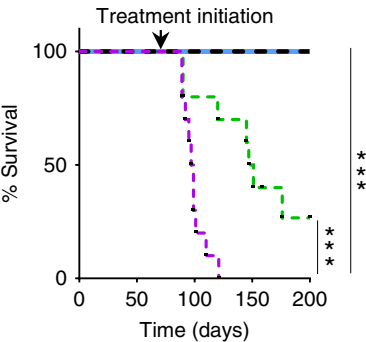

b

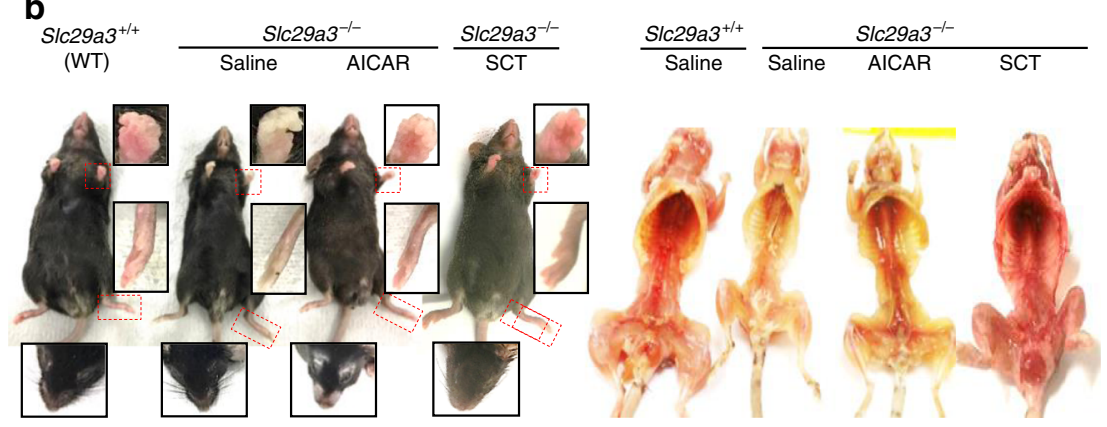

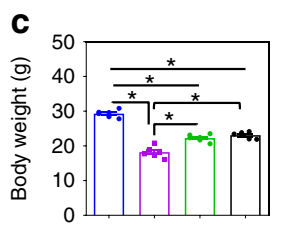
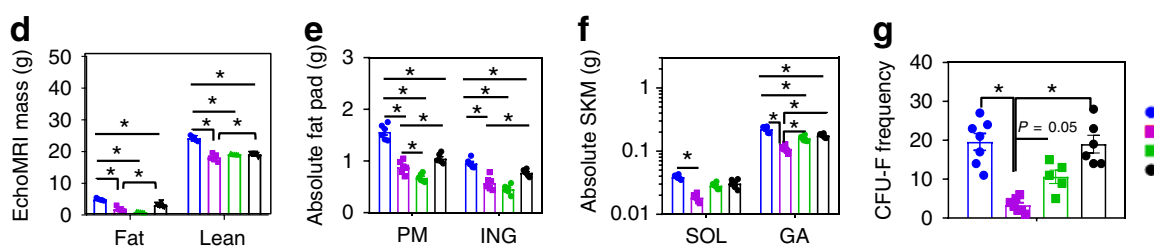

- $\mathrm{SIc}_{29 a 3^{++}}$(WT) SIC29a3-
Slc29a3
$-1-$

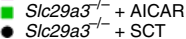

h i

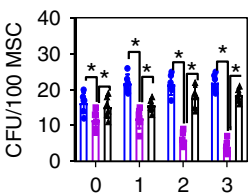

i

\section{j}
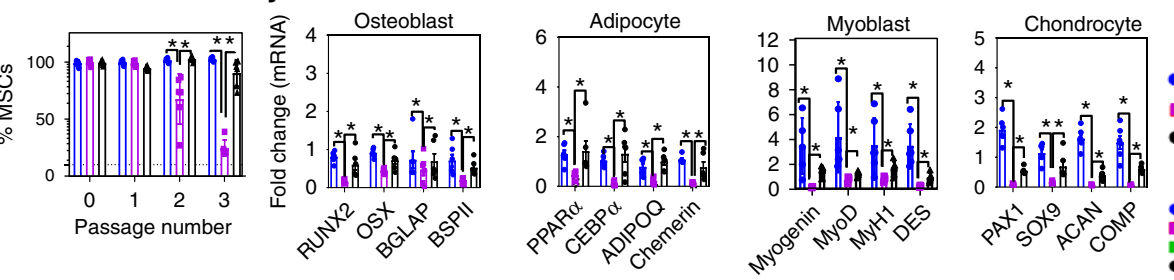

- $\mathrm{Slc}_{29 \mathrm{a} 3^{+/+}}$(WT)

SIc29a $3^{-/-}$control

Passage number

- Slc29a3 $3^{+/+}$(WT)

SIc29a3 $3^{-1-}+$ AICAR

k
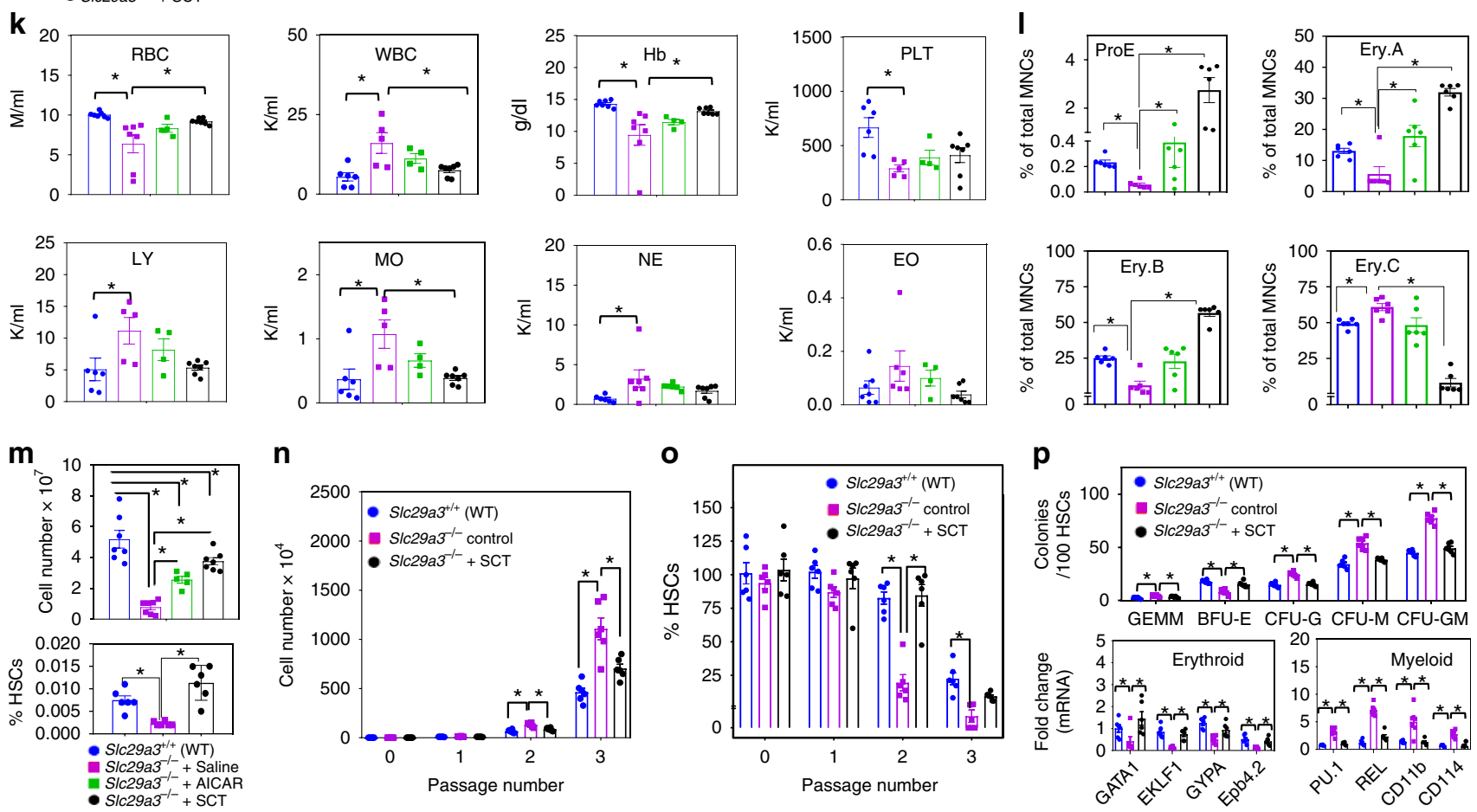

n

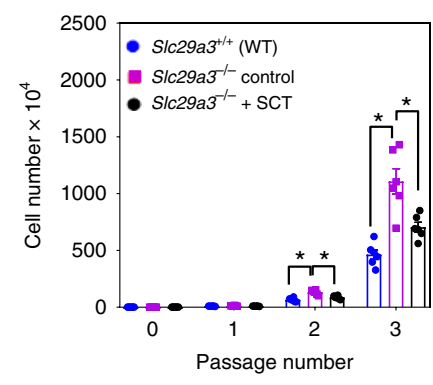

o

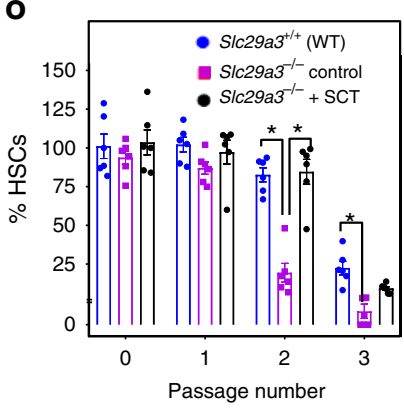

p

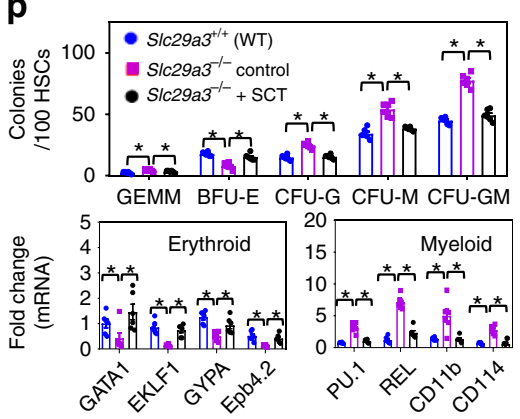

Maintenance of Slc29a3 ${ }^{-/-}$mice. All animal procedures were performed according to protocols approved by the Ohio State University (OSU) IACUC. The Slc29a3 $3^{+/}$gene trap knockout line in a 129 S5/SvEvBrd x C57BL6/J hybrid background was obtained from the Mutant Mouse Resource and Research Center. A heterozygote breeding strategy was utilized to generate Slc29a3 ${ }^{+/+}, \operatorname{Slc} 29 a 3^{+/-}$and Slc29a3 ${ }^{-1}$ - littermates. In general, F1 HET mice were crossed to produce F2 WT, HET, and HOM null cohorts, and phenotypic analyses were conducted on 3-12 animals of either sex (unless otherwise stated) in age groups 8-16 weeks as indicated. In addition to littermate WT, HOM null cohorts were also compared to WT reference controls $(\mathrm{C} 57 \mathrm{BL} / 6 \mathrm{NCrl})$ as needed. When HOM null male and female animals were crossed, several breeding pairs were set up to obtain viable litters. For genotyping, tail clips were obtained at 3 weeks of age, and DNA was isolated by proteinase $\mathrm{K}$ digestion followed by the isopropanol/ethanol precipitation method. Genotyping was performed by PCR using the following primers: WT forward: GCCAGAGGATCGCTTCA; WT reverse: GACAACAGCAGGCAATCTAAA; KO forward: ATGCAAATGGCCTCCCTCAAGTACC; KO reverse: GAGGAAATTG CATCGCATTGTCT primers. The PCR cycling conditions to generate product sizes of 415 and $284 \mathrm{bp}$, for WT and KO, respectively, were as follows: WT: 
Fig. 9 AICAR and SCT improve survival and alleviate dysfunction in S/c29a3-/- mice. AICAR injection (500 mg/kg; SID) ( $n=10 / \mathrm{group})$ and SCT (1 $\times 10^{4}$

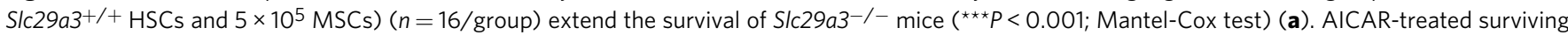
mice show facial alopecia (insets; below), while both the AICAR and SCT groups show improved appearance and medullary hematopoiesis (right) (b). Changes in body weight (c), EchoMRI-measured fat and lean mass (d), absolute parametrial (PM) and inguinal (ING) fat pad mass (e), absolute soleus ( $\mathrm{SOL}$ ) and gastrocnemius (GA) skeletal muscle (SKM) mass (f) and bone marrow CFU-F frequency (g) in AICAR-treated surviving mice and SCT mice $(n=6$, mean \pm SEM). Clonogenicity $(\mathbf{h})$ and marker expression (i) with serial passage of MSCs derived from SCT mice $(n=6$, mean \pm SEM). mRNA expression of transcription factors and markers after 14 (osteoblasts and adipocytes) and 28 (myocytes and chondrocytes) days of the differentiation of MSCs derived from SCT mice $(n=6$, mean \pm SEM) $(\mathbf{j})$. Hematological parameters in AICAR-treated surviving mice and SCT mice. AICAR-treated surviving S/c29a3- - - mice $(n=4$, mean \pm SEM $)$ and SCT mice $(n=7$, mean \pm SEM $)$ at 28 weeks are compared with saline-treated S/c29a3 $-/-(n=5$, mean \pm SEM $)$ and WT mice $(n=6$, mean \pm SEM) at 12 weeks $(\mathbf{k})$. Frequency of erythroid subpopulations within the bone marrow of AICAR-treated surviving mice and SCT mice $(n=6$, mean \pm SEM) (I). Cellularity (above) and HSC frequencies (below) in AICAR-treated $(n=5$, mean \pm SEM) and SCT mouse bone marrow $(n=7$, mean \pm SEM $)(\mathbf{m})$. Culture expansion capacity $(\mathbf{n})$ and percent of cells expressing HSC markers (o) with serial passage of SCT mouse bone marrowderived HSCs $(n=6$, mean \pm SEM). CFU-forming capacity (above) and mRNA expression of transcription factors and markers (below) after 14 days of HSC differentiation in SCT mice $(n=6$, mean \pm SEM) (p). SCT, stem cell transplantation; CFU, colony-forming unit; CFU-F, CFU-Fibroblast; Hb, hemoglobin; PLT, platelet; LY, lymphocyte; MO, monocyte; NE, neutrophil; EO, eosinophil; ProE, proerythroblasts; EryA, early basophilic erythroblasts; EryB, late basophilic and polychromatic erythroblasts; EryC, orthochromatic erythroblasts/reticulocytes; GEMM, granulocyte, erythrocyte, monocyte, megakaryocyte; BFU-E, burst-forming unit-erythroid, colony-forming unit, CFU; CFU-M, CFU-macrophage, CFU-G, CFU-granulocyte; CFU-GM, CFUgranulocyte, macrophage. Statistical analyses were performed using ANOVA with Tukey's multiple comparisons post-test and two-tailed Student's $t$-test. ${ }^{\star} P<0.05$. Source data are provided as a Source Data file

denaturation at $94^{\circ} \mathrm{C}$ for $2 \mathrm{~min}$; four cycles of $94^{\circ} \mathrm{C}$ for $20 \mathrm{~s}, 62^{\circ} \mathrm{C}$ for $20 \mathrm{~s}$, and $72{ }^{\circ} \mathrm{C}$ for $20 \mathrm{~s} ; 36$ cycles of $94^{\circ} \mathrm{C}$ for $20 \mathrm{~s}, 55^{\circ} \mathrm{C}$ for $20 \mathrm{~s}$, and $72{ }^{\circ} \mathrm{C}$ for $20 \mathrm{~s}$; and final extension at $72^{\circ} \mathrm{C}$ for $1 \mathrm{~min}$; KO: denaturation at $94^{\circ} \mathrm{C}$ for $2 \mathrm{~min}$; 40 cycles of $94^{\circ} \mathrm{C}$ for $10 \mathrm{~s}, 58^{\circ} \mathrm{C}$ for $20 \mathrm{~s}$, and $72^{\circ} \mathrm{C}$ for $20 \mathrm{~s}$; and final extension at $72{ }^{\circ} \mathrm{C}$ for $1 \mathrm{~min}$ for KO. All the mice were maintained at an ambient temperature of $20-22^{\circ} \mathrm{C}$ with a 12-h light/dark cycle and were given free access to standard rodent chow and water.

Clinical phenotyping of Slc29a3 ${ }^{-/-}$mice. The animals were acclimated to the motorized treadmill (model Eco3/6; Columbus Instruments) by running for $5 \mathrm{~min}$ at $8 \mathrm{~m} / \mathrm{min}$ each day for 3 days. On the day of experimentation, an electrical stimulus $(163 \mathrm{~V}, 0.45-\mathrm{mA}$ current, $1 \mathrm{~Hz})$ was applied using electrical shock grids at the rear end of the treadmill that allowed the animals to exercise until exhaustion. The inability to continue treadmill running at treadmill exhaustion was used to evaluate the endurance capacity.

MicroCT images of the tibia were acquired at $80 \mathrm{kVp}$ and $490 \mathrm{~mA}$ using a General Electric Locus RS (General Electric HealthCare, UK). The voxel size was $0.020367 \times 0.020367 \times 0.020367 \mathrm{~mm}$. For image analysis, a region-of-interest $(\mathrm{ROI})$ that included $0.3055 \mathrm{~mm}$ above the growth plate was used for trabecular analysis. The trabecula was segmented using a locally adaptive segmentation algorithm. The percent bone and percent marrow were calculated from the segmented trabecula image $^{61}$. The trabecular thickness, spacing, and number were calculated from the Euclidean distance map of the segmented trabecula ${ }^{61,62}$. Bone mineral density measurements for the trabecular region and two cortical regions (approximately 2.67 , and $6.5 \mathrm{~mm}$ from the growth in the diaphysis of the bone) were calculated using a hydroxyapatite calibration phantom.

The mice were subjected to non-invasive monitoring of whole-body lean and fat mass by Echo MRI at the Small Animal Imaging Facility, OSU63.

For bone marrow transplantation experiments, recipient WT or Slc29a3-1mice were given total body irradiation with 9.5 Gy using an RS $2000 \times$-ray irradiator or 10.5 Gy using a Small Animal Radiation Research Platform (SARRP) system. After irradiation, $1 \times 10^{4}$ bone marrow-derived $\mathrm{Lin}^{-} \mathrm{Scal}^{+}{ }^{+} \mathrm{c}_{\text {-kit }}{ }^{+}$FLT3CD34+ WT or Slc29a3-l- HSCs in $100 \mu$ of PBS were injected intravenously into the tail vein (details in Supplementary Methods). The mice were monitored for survival over a period of 15 days. The Kaplan-Meier survival plot was created based on the estimation of survival data. Whole bone marrow was collected at the end of 15 days (or at the day of mortality), and the number of bone marrow cells per limb was assessed.

\section{Lysosome isolation and analyte quantification using liquid}

chromatography-mass spectrometry (LC-MS/MS). Enriched lysosomes from the mouse spleen and HEK293 cells were obtained by differential centrifugation, followed by density gradient centrifugation and calcium precipitation (LYSISO1; Sigma-Aldrich, St. Louis, MO). To determine the enrichment and recovery of lysosomes, the total homogenate and different lysosomal fractions were compared for the protein concentration (using BCA protein assay) and acid phosphatase activity (CS0740; Sigma-Aldrich, St. Louis, MO), while the intactness of the lysosomes was assessed using the neutral red dye following manufacturer's protocol (CS0740; Sigma-Aldrich, St. Louis, MO).

The extraction of adenosine from the purified lysosomal fractions and adenosine and AMP from the cytosolic fractions was performed using $100 \mu \mathrm{l}$ of cold acetonitrile: methanol $(3: 1, \mathrm{v} / \mathrm{v})$ spiked with reserpine $(1 \mu \mathrm{g} / \mathrm{ml})$ as the internal standard. The samples were vortex mixed and then were centrifuged at 15,000 rpm at $4{ }^{\circ} \mathrm{C}$ for $10 \mathrm{~min}$. The supernatant was filled in auto-sampler vials, and volumes of $10 \mu \mathrm{l}$ were injected into an LC-MS/MS system. LC-MS/MS analysis was performed using a Thermo Scientific Vanquish UPLC system (Waltham, MA) interfaced with a Thermo Scientific TSQ Quantiva triple-stage quadrupole mass spectrometer (Waltham, MA) equipped with an H-ESI ion source controlled by Thermo XcaliburTM 3.0 data acquisition and analysis software. MS detection was carried out in the positive ionization mode, and the transitions monitored were $\mathrm{m} / z 268.12$ to 136.11 for adenosine, 348.13 to 136.11 for AMP, 259.00 to 136.11 for AICAR and 609.39 to 195.05 for reserpine. The operational mass spectrometric parameters included the following: capillary voltage, $4.5 \mathrm{kV}$; sheath gas, 35 arbitrary units; auxiliary gas, 10 arbitrary units; sweep gas, 2 arbitrary units; ion transfer tube temperature, $350{ }^{\circ} \mathrm{C}$; vaporizer temperature, $450{ }^{\circ} \mathrm{C}$; Dwell time, $50 \mathrm{~ms}$ per transition; collision-induced dissociation (CID) gas, 1.5 mtorr. The collision energy was set at $20 \mathrm{~V}$ for adenosine, AICAR, and AMP, and at $38 \mathrm{~V}$ for reserpine. Chromatographic separation was carried out using a Scherzo $\mathrm{SMC}_{18}$ column $(150 \times 4.6 \mathrm{~mm} ; 3-\mu \mathrm{m}$ particle size $)$ from Imtakt (Portland, OR). The mobile phase consisted of solvent A [water ( $0.1 \%$ formic acid)] and solvent B [acetonitrile $(0.1 \%$ formic acid)]. The flow rate was set at $0.5 \mathrm{ml} / \mathrm{min}$, and the gradient program used was 0 min: $95 \%$ B; 2 min: $95 \%$ B; 10 min: $10 \%$ B; 11 min: $95 \%$ B; 17 min $95 \%$ B. The total run time was $17 \mathrm{~min}$ per sample, and the auto-sampler was maintained at $4{ }^{\circ} \mathrm{C}$ throughout the analysis.

Untargeted and targeted metabolomics. Metabolites were extracted using a liquid-liquid extraction (LLE) method and a mixture of methanol/chloroform/ hexane $(2 / 0.5 / 0.5, v / v / v)$. Further analyses were carried out using a Thermo Scientific LTQ Orbitrap ${ }^{\text {Ts }}$ Hybrid Ion Trap-Orbitrap mass spectrometer (MS) and a Waters 2795 HPLC separation module interfaced by an electrospray ionization (ESI) source. The metabolites were separated on a Scherzo $\mathrm{SMC}_{18}$ column (150 $\mathrm{mm} \times 4.6 \mathrm{~mm}$; $3-\mu \mathrm{m}$ particle size; Imtakt, Portland, OR). The mobile phase used for chromatographic separation was as follows: solvent $\mathrm{A}, 10 \mathrm{mM}$ ammonium acetate:acetonitrile $(40: 60, v / v)$; solvent B, 2-propanol:acetonitrile $(90: 10, v / v)$. The optimized chromatographic gradient conditions were as follows: $0 \mathrm{~min}, 95 \% \mathrm{~A} ; 2$ $\min , 95 \%$ A; $22 \mathrm{~min}, 10 \%$ A; $27 \mathrm{~min}, 10 \%$ A; $28 \mathrm{~min}, 95 \%$ A; $35 \mathrm{~min}, 95 \%$ A; the flow rate was set at $0.5 \mathrm{ml} / \mathrm{min}$. Data acquisition and analysis were conducted using Thermo Xcalibur ${ }^{\mathrm{TM}}$ software. The data were collected separately in the positive and negative electrospray ionization modes, and the acquisition was performed in the centroid mode using the full-scan MS method. The general mass spectrometer operating conditions were as follows: Spray voltage: $+4.5 \mathrm{kV}$ for the positive mode and $-3.5 \mathrm{kV}$ for the negative mode; source temperature: $350{ }^{\circ} \mathrm{C}$; vaporizer temperature: $450{ }^{\circ} \mathrm{C}$; sheath gas: 35 arbitrary units; auxiliary gas: 10 arbitrary units; sweep gas: 2 arbitrary units; mass scan range: $\mathrm{m} / \mathrm{z} 50-1200$ at $0.1 \mathrm{scan} / \mathrm{s}$.

For MS/MS analysis, the mass parameters were as follows: isolation width: 2.0 $(\mathrm{m} / \mathrm{z})$; collision-induced dissociation (CID): 40\%; resolution: 30,000; maxIT: 100 ms. An open source software package MZmine v.2.21 (http://mzmine.sourceforge. net/) was used for data processing. The RANSAC algorithm was applied to align the detected peaks in the different samples, generating an aligned peak list for Slc29a3 $3^{+1+} \& S l c 29 a 3^{-1-}$ samples. A database search using the Human Metabolome Database (HMDB) (http://www.hmdb.ca), KEGG pathway database (http://www.genome.jp/kegg/) \& Lipid Metabolites and Pathway Strategy (Lipid Maps) (http://www.lipidmaps.org) was performed to establish peak identities for each $\mathrm{m} / z$ in the aligned peak list with a mass tolerance set to $\pm 10 \mathrm{ppm}$. Lipidomics analysis of the liver samples from Slc29a3 ${ }^{+1+}$ \& Slc29a3 ${ }^{-1-}$ was performed using the method described by Park et al. ${ }^{64}$ 


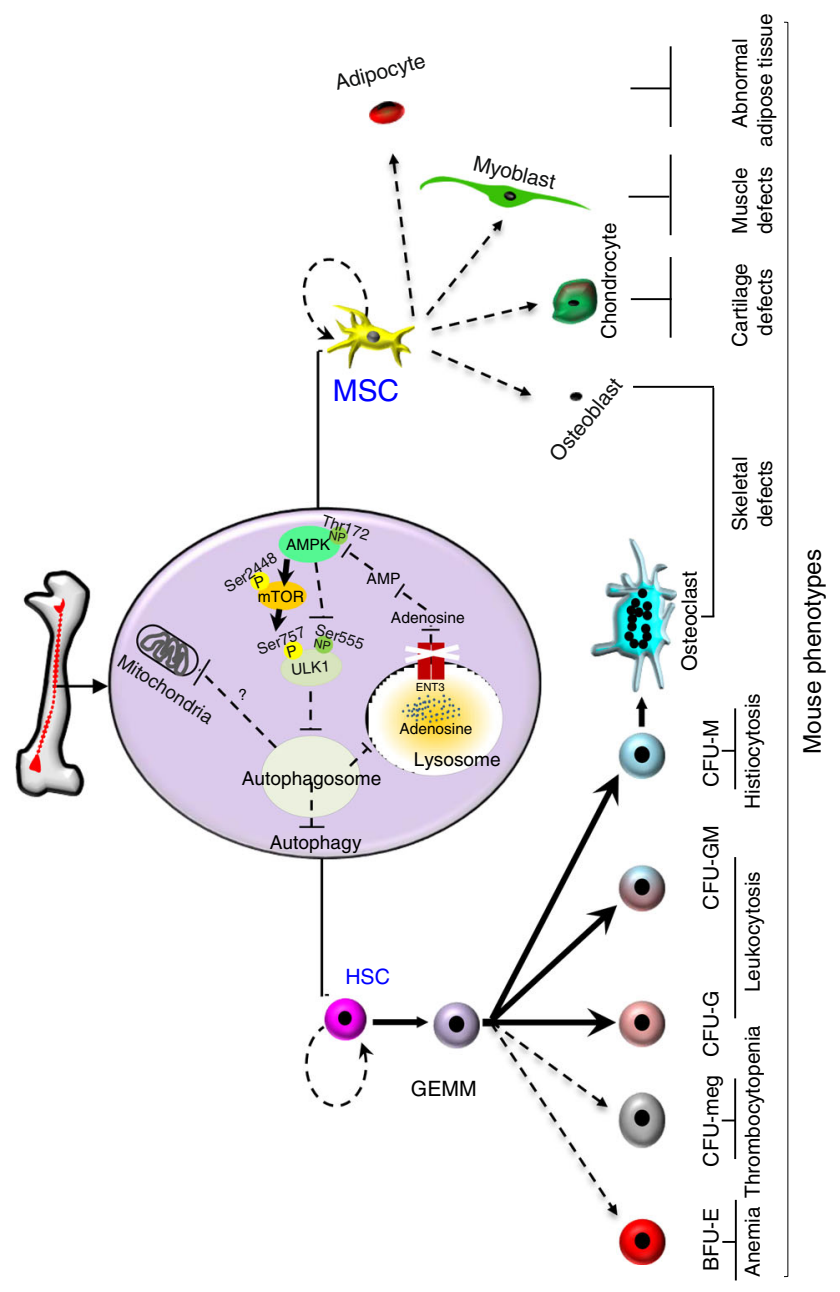

Fig. 10 Schematic representation of disease pathogenesis in S/c29a3-/mice. Molecular, cellular and tissue defects in S/c29a3-/- mice are depicted. Broken lines or arrows indicate inhibitory pathways, and thick arrows indicate the stimulatory pathways occurring in Slc29a3-/- mice. Phosphorylation statuses of AMPK, mTOR, and ULK in S/c29a3-/- cells are shown ( $P$, phosphorylated, yellow circle; NP, non-phosphorylated, green circle). CFU, colony-forming unit; E, erythroid; meg, megakaryocyte; G, granulocyte; $M$, macrophage; GM, granulocyte/macrophage; GEMM, granulocyte/erythrocyte/macrophage/megakaryocyte

Functional enrichment of the metabolome data with KEGG pathway annotations was performed using MBROLE2 ${ }^{65}$ (http://csbg.cnb.csic.es/mbrole2/). Integrated pathway analysis of transcriptome and metabolome data was performed using MetaboAnalyst ${ }^{\circ} 3.0^{66}$ by employing the KEGG pathway database. The integrated metabolomics and transcriptomics network was constructed based on the HMDB ID for metabolites and gene symbols for transcripts using the open source software $\mathrm{GAM}^{67}$ (https://artyomovlab.wustl.edu/shiny/gam), ipath ${ }^{68}$, and LRpath ${ }^{69}$.

Transmission electron microscopy. Liver sections from animals or MSCs were fixed with $2 \%$ glutaraldehyde for $3 \mathrm{~h}$. After washing in phosphate buffer $(0.1 \mathrm{M})$, the tissues were processed for secondary fixation with $1 \%$ osmium tetroxide for $1 \mathrm{~h}$, followed by ethanol dehydration, infiltration, and embedding in Eponate 12 resin for ultrathin sectioning using a Reichert Ultracut E ultramicrotome. The sections were stained using $2 \%$ aqueous uranyl acetate followed by Reynolds lead citrate and observed by TEM using FEI Technai Spirit electron microscope at $80 \mathrm{kV}$. For scoring autophagosomes and/or lysosomes in TEM studies, slide identities were blinded and predefined stereoptic criteria were used to quantify structures of interest.

Immunocytochemical analysis. Cells were plated on coverslips in six-well clusters and were allowed to grow until they were $60-80 \%$ confluent. The cells were fixed with $4 \%$ paraformaldehyde (PFA), rinsed with PBS, permeabilized with $0.1 \%$ Triton X-100, and incubated in a standard blocking solution (10\% donkey serum,
$1 \%$ BSA in PBS) for $1 \mathrm{~h}$. The cells were then incubated overnight at $4{ }^{\circ} \mathrm{C}$ with primary antibody at a 1:200 to 1:1000 dilution. Subsequently, the cells were washed with PBS and incubated for $1 \mathrm{~h}$ with a 1:1000 dilution of Alexa Fluor 647 goat antirabbit IgG (Invitrogen) or Alexa Fluor 647 donkey anti-Goat IgG (Invitrogen) secondary antibody, respectively. Nuclei were stained with DAPI (Thermo Fisher Scientific) and examined using an Olympus Spectral FV1000 confocal microscope or Nikon ti2 inverted fluorescence microscope.

Gene expression analysis. Total RNA was extracted from cells using the E.Z.N.A Total RNA Kit (Omega Biotek, Norcross, GA) following the manufacturer's protocol, and the RNA integrity was measured using the Agilent 2100 Bioanalyzer (Agilent Technologies, Palo Alto, CA). One microgram of total RNA was reverse transcribed using the RevertAid RT Reverse Transcription kit (Thermo Fisher Scientific, Waltham, MA). Quantitative PCR was performed using a StepOnePlus Real-Time PCR System (Applied Biosystems, CA, USA). The Ct values for GAPDH and GusB were used to normalize the expression level of the gene of interest using the $\Delta \Delta C T$ method $^{70}$. The PrimerBank web application was used to design the exon-spanning primers listed below.

BSPII-F: GACTTTTGAGTTAGCGGCACT; BSPII-R: CCGCCAGCTCGTTTTCATC; BGLAP-F: CTGACCTCACAGATCCCAAGC; BGLAP-R: TGGTCTGATAGCTCGTCACAAG; OSX-F: ACCCCAAGATGTCTATAAGCCC; OSX-R: CGCTCTAGCTCCTGACAGTTG; Runx2-F: GACTGTGGTTACCGTCATGGC; Runx2-R:

ACTTGGTTTTTCATAACAGCGGA; PPAR $\gamma$-F:

TCGCTGATGCACTGCCTATG; PPAR $\gamma$-R: GAGAGGTCCACAGAGCTGATT; CEBP $\alpha$-F: GCGGGAACGCAACAACATC; CEBP $\alpha-R$

GTCACTGGTCAACTCCAGCAC; Adiponectin- F: AGCCGCTTATATGTATCGCTCA; Adiponectin-R: TGCCGTCATAATGATTCTGTTGG Chemerin-F: TTGCTGATCTCCCTAGCCCTA; Chemerin-R: TGGGTGTTTGTGGAACTCCTC; MyoD-F: GACAGGGAGGAGGGGTAGAG; MyoD-R: TGCTGTCTCAAAGGAGCAGA; Myogenin-F: CCGAGCCTCTACAACAGGAG; Myogenin-R: GGTAACTTCCTCACCCACGA MYH1-F: GCGAATCGAGGCTCAGAACAA; MYH1-R: GTAGTTCCGCCTTCGGTCTTG; DES-F: CCTGGAGCGCAGAATCGAAT; DES-R: TGAGTCAAGTCTGAAACCTTGGA; SLC29A3-F:

GGGCATATAAACTCCGAAACTGC; SLC29A3-R

GGAGGAAGTATCCACCTTCACC; PAX1-F: CCGCCTACGAATCGTGGAG; PAX1-R: CCCGCAGTTGCCTACTGATG; SOX9-F: AGTACCCGCATCTGCACAAC; SOX9-R: ACGAAGGGTCTCTTCTCGCT; ACAN-F: GTGGAGCCGTGTTTCCAAG; ACAN-R:

AGATGCTGTTGACTCGAACCT; COMP-F: ACTGCCTGCGTTCTAGTGC; COMP-R: CGCCGCATTAGTCTCCTGAA; GATA1-F

TGTCCTCACCATCAGATTCCA; GATA1-R: TCCCTCCATACTGTTGAGCAG; EKLF1-F: TCTGAGGAGACGCAGGATTTG; EKLF1-R:

ACAGGTCACGTCCCTCTCATC; GYPA-F:

TACCAAGAAGAGCATTCACCATC; GYPA-R:

TGCTGATTTGGGTTACCTACAGT; Epb4.2-F:

CCACGCAGCAGAAAACAACG; Epb4.2-R: GAGCACGGAAGTTCAGGGT; PU.1-F: TTACAGGCGTGCAAAATGGAA; PU.1-R: GACGTTGGTATAGCTCTGAATCG; REL-F: AGAGGGGAATGCGGTTTAGAT; REL-R: TGTCCGGTTGTTGTCTGTGC: CD11b-F: CCATGACCTTCCAAGAGAATGC; CD11b-R: ACCGGCTTGTGCTGTAGTC; CD14-F: ACTTCTCAGATCCGAAGCCAG; CD14-R: CCGCCGTACAATTCCACAT; LGR5-F:

ACATTCCCAAGGGAGCGTTC; LGR5-R: ATGTGGTTGGCATCTAGGCG; EPHB3-F: CTGTGCGTGCCTTCTACAAGA; EPHB3-R:

GCTTGAGTGGTACAGAGACCTC; AXIN2-F:

AACCTATGCCCGTTTCCTCTA; AXIN2-R:

GAGTGTAAAGACTTGGTCCACC; LEF1-F.

CCAGAGAACACCCTGATGAAGG; LEF1-R:

GGCACTTTATTTGATGTCCTCGG; HES1-F:

TCAACACGACACCGGACAAAC; HES1-R: ATGCCGGGAGCTATCTTTCTT; HEY1-F: CCGACGAGACCGAATCAATAAC; HEY1-R: TCAGGTGATCCACAGTCATCTG; HEY2-F: GAGGTCCAATTCACCGACAAC; HEY2-R: AGCATGGGCATCAAAGTAGCC; NRARP-F: TTCAACGTGAACTCGTTCGGG; NRARP-R: TTGCCGTCGATGACTGACTG; REX1-F: AAGCTGCCAGCCAGTAACC; REX1-R:

CCTTGCGTTCCACCAACTTTC; TERT-F:

CTGAGTCTCACCAGTACAAGTGT; TERT-R: TTGGCACCCATGATTTGCCT; NANOG-F: AGGACAGGTTTCAGAAGCAGA; NANOG-R: CCATTGCTAGTCTTCAACCACTG; SOX2-F: CCCACCTACAGCATGTCCTAC; SOX2-R: GCCTCGGACTTGACCACAG; GAPDH-F: TGGCCTTCCGTGTTCCTAC; GAPDH-R: GAGTTGCTGTTGAAGTCGCA

Statistical analyses. Comparisons between values were performed using Student's $t$ test (two-tailed), unless otherwise indicated, after confirming that the data met appropriate assumptions (normality, homogenous variance, and independent sampling). All comparisons between multiple groups were performed using one-way or 
two-way analysis of variance (ANOVA) with Tukey's post-hoc analysis. For all statistical analyses, $P<0.05$ was considered statistically significant, unless otherwise indicated. The results are expressed as the means \pm SEM, unless otherwise mentioned.

Multivariate statistical analysis was performed for the identified metabolite peak lists using MetaboAnalyst ${ }^{\oplus}$ 3.0. Principal component analysis (PCA), partial least squares discriminant analysis (PLS-DA) and orthogonal projection to latent structures discriminant analysis (OPLS-DA) were used to visualize clustering of the Slc29a3 $3^{+/+}$\& Slc29a3 $3^{-1-}$ samples. Unsupervised hierarchical clustering for data overview was performed using a heatmap generated using a $t$-test, the Euclidean distance measurement, and the Ward clustering algorithm.

For all in vitro experiments, a sample size of $n=3-12$ was used based on the effect size and overlap between distributions. A power of 0.8 was set as minimal to decide sample size for each experiment. For the in vivo studies that assessed the effects of AICAR treatment or stem cell transplant on survival of Slc29a3 ${ }^{-1-}$ mice, a sample size of $n=10-16$ independent mice per group was used to include inherent variabilities in survival times among Slc29a3-l- mice. Sample sizes were estimated using power calculations for guidance (http://biomath.info/power/ ttest.htm) with effect sizes adjudged from pilot studies. With alpha $=0.05$ and power $=0.9$ and allowing for some unexpected mortalities ( $\sim 10 \%$ mouse/group $), \geq$ 7-8 mice per group were needed for in vivo studies. For in vivo bone marrow transplant mice study, a sample size of $n=6$ independent mice per group was set because a definitive end point mortality within 7 days post irradiation was achieved in all nontransplanted mice. The sample size for the metabolomics and lipidomics experiments was calculated based on the power analysis module in Metaboanalyst software 66 , which uses algorithms described by van Iterson et al. ${ }^{71}$. The desired power of 0.8 was achieved for both the metabolomics and lipidomics data validating a sample size of $n=12$ independent mice per group. No animals or samples were excluded from the analyses.

Reporting summary. Further information on research design is available in the Nature Research Reporting Summary linked to this article.

\section{Data availability}

The data sets generated during and/or analyzed during the current study are available within the article and its Supplementary Information files, or from the corresponding author on reasonable request. A reporting summary for this Article is available as a Supplementary Information file. The source data underlying Figs. 1-9 and Supplementary Figs. 4-7, 10 and 12 are provided as a Source Data file. Metabolomics and lipidomics data have been deposited to the EMBL-EBI MetaboLights database (https:// doi.org/10.1093/nar/gks1004. PubMed PMID: 23109552) with the identifier MTBLS949.

Received: 22 December 2017 Accepted: 7 June 2019

Published online: 03 July 2019

\section{References}

1. Rossbach, H. C., Dalence, C., Wynn, T. \& Tebbi, C. Faisalabad histiocytosis mimics Rosai-Dorfman disease: brothers with lymphadenopathy, intrauterine fractures, short stature, and sensorineural deafness. Pedia. Blood Cancer 47, 629-632 (2006).

2. Zur Stadt, U. et al. Mutation spectrum in children with primary hemophagocytic lymphohistiocytosis: molecular and functional analyses of PRF1, UNC13D, STX11, and RAB27A. Hum. Mutat. 27, 62-68 (2006).

3. Prendiville, J. et al. Pigmented hypertrichotic dermatosis and insulin dependent diabetes: manifestations of a unique genetic disorder? Pedia. Dermatol 24, 101-107 (2007).

4. Hussain, K. et al. Diabetes mellitus, exocrine pancreatic deficiency, hypertrichosis, hyperpigmentation, and chronic inflammation: confirmation of a syndrome. Pedia. Diabetes 10, 193-197 (2009).

5. Molho-Pessach, $\mathrm{V}$. et al. The $\mathrm{H}$ syndrome is caused by mutations in the nucleoside transporter hENT3. Am. J. Hum. Genet. 83, 529-534 (2008).

6. Cliffe, S. T. et al. SLC29A3 gene is mutated in pigmented hypertrichosis with insulin-dependent diabetes mellitus syndrome and interacts with the insulin signaling pathway. Hum. Mol. Genet. 18, 2257-2265 (2009).

7. Morgan, N. V. et al. Mutations in SLC29A3, encoding an equilibrative nucleoside transporter ENT3, cause a familial histiocytosis syndrome (Faisalabad histiocytosis) and familial Rosai-Dorfman disease. PLoS Genet. 6, e1000833 (2010).

8. Priya, T. P. et al. H syndrome: novel and recurrent mutations in SLC29A3. Br. J. Dermatol. 162, 1132-1134 (2010).

9. Ramot, Y. et al. Early-onset sensorineural hearing loss is a prominent feature of $\mathrm{H}$ syndrome. Int J. Pedia. Otorhinolaryngol. 74, 825-827 (2010).

10. Campeau, P. M. et al. Whole-exome sequencing identifies mutations in the nucleoside transporter gene SLC29A3 in dysosteosclerosis, a form of osteopetrosis. Hum. Mol. Genet. 21, 4904-4909 (2012).
11. Colmenero, I., Molho-Pessach, V., Torrelo, A., Zlotogorski, A. \& Requena, L. Emperipolesis: an additional common histopathologic finding in $\mathrm{H}$ syndrome and Rosai-Dorfman disease. Am. J. Derm. 34, 315-320 (2012).

12. Farooq, M. et al. Identification of two novel mutations in SLC29A3 encoding an equilibrative nucleoside transporter (hENT3) in two distinct Syrian families with H syndrome: expression studies of SLC29A3 (hENT3) in human skin. Dermatology 224, 277-284 (2012).

13. de Jesus, J. et al. SLC29A3 mutation in a patient with syndromic diabetes with features of pigmented hypertrichotic dermatosis with insulin-dependent diabetes, $\mathrm{H}$ syndrome and Faisalabad histiocytosis. Diabetes Metab. 39, 281-285 (2013).

14. Elbarbary, N. S. et al. An Egyptian family with $\mathrm{H}$ syndrome due to a novel mutation in SLC29A3 illustrating overlapping features with pigmented hypertrichotic dermatosis with insulin-dependent diabetes and Faisalabad histiocytosis. Pedia. Diabetes 14, 466-472 (2013).

15. Molho-Pessach, V. et al. H syndrome: the first 79 patients. J. Am. Acad. Dermatol. 70, 80-88 (2014).

16. Al-Haggar, M. et al. Novel homozygous SLC29A3 mutations among two unrelated Egyptian families with spectral features of $\mathrm{H}$-syndrome. Pedia. Diabetes 16, 305-316 (2015).

17. Young, J. D., Yao, S. Y., Baldwin, J. M., Cass, C. E. \& Baldwin, S. A. The human concentrative and equilibrative nucleoside transporter families, SLC28 and SLC29. Mol. Asp. Med. 34, 529-547 (2013).

18. Kang, N. et al. Human equilibrative nucleoside transporter-3 (hENT3) spectrum disorder mutations impair nucleoside transport, protein localization, and stability. J. Biol. Chem. 285, 28343-28352 (2010).

19. Baldwin, S. A. et al. Functional characterization of novel human and mouse equilibrative nucleoside transporters (hENT3 and mENT3) located in intracellular membranes. J. Biol. Chem. 280, 15880-15887 (2005).

20. Govindarajan, R. et al. Facilitated mitochondrial import of antiviral and anticancer nucleoside drugs by human equilibrative nucleoside transporter-3. Am. J. Physiol. Gastrointest. Liver Physiol. 296, G910-G922 (2009).

21. Hsu, C. L. et al. Equilibrative nucleoside transporter 3 deficiency perturbs lysosome function and macrophage homeostasis. Science 335, 89-92 (2012).

22. Rahman, M. F., Askwith, C. \& Govindarajan, R. Molecular determinants of acidic $\mathrm{pH}$-dependent transport of human equilibrative nucleoside transporter3. J. Biol. Chem., https://doi.org/10.1074/jbc.M117.787952 (2017).

23. Singh, A. \& Govindarajan, R. ENT3 utilizes a $\mathrm{pH}$ sensing mechanism for transport. Channels (Austin), https://doi.org/10.1080/19336950.2017.1389581 (2017).

24. Bolze, A. et al. A mild form of SLC29A3 disorder: a frameshift deletion leads to the paradoxical translation of an otherwise noncoding mRNA splice variant. PLoS ONE 7, e29708 (2012).

25. Melki, I. et al. Mutation in the SLC29A3 gene: a new cause of a monogenic, autoinflammatory condition. Pediatrics 131, e1308-e1313 (2013).

26. Biteau, B., Hochmuth, C. E. \& Jasper, H. Maintaining tissue homeostasis: dynamic control of somatic stem cell activity. Cell Stem Cell 9, 402-411 (2011)

27. Kaufman, D. S., Hanson, E. T., Lewis, R. L., Auerbach, R. \& Thomson, J. A Hematopoietic colony-forming cells derived from human embryonic stem cells. Proc. Natl Acad. Sci. USA 98, 10716-10721 (2001).

28. Zhang, P. et al. Negative cross-talk between hematopoietic regulators: GATA proteins repress PU.1. Proc. Natl Acad. Sci. USA 96, 8705-8710 (1999).

29. Guo, L., Zhou, Y., Wang, S. \& Wu, Y. Epigenetic changes of mesenchymal stem cells in three-dimensional (3D) spheroids. J. Cell Mol. Med 18, 2009-2019 (2014)

30. Han, J. et al. Nanog reverses the effects of organismal aging on mesenchymal stem cell proliferation and myogenic differentiation potential. Stem Cells 30, 2746-2759 (2012)

31. Garcia-Prat, L. et al. Autophagy maintains stemness by preventing senescence. Nature 529, 37-42 (2016).

32. Settembre, C. et al. TFEB links autophagy to lysosomal biogenesis. Science 332, 1429-1433 (2011)

33. Deter, R. L., Baudhuin, P. \& De Duve, C. Participation of lysosomes in cellular autophagy induced in rat liver by glucagon. J. Cell Biol. 35, C11-C16 (1967).

34. Komatsu, M. et al. Homeostatic levels of p62 control cytoplasmic inclusion body formation in autophagy-deficient mice. Cell 131, 1149-1163 (2007).

35. Klionsky, D. J. et al. Guidelines for the use and interpretation of assays for monitoring autophagy (3rd edition). Autophagy 12, 1-222 (2016).

36. Mortensen, M. et al. Loss of autophagy in erythroid cells leads to defective removal of mitochondria and severe anemia in vivo. Proc. Natl Acad. Sci. USA 107, 832-837 (2010).

37. Mortensen, M., Watson, A. S. \& Simon, A. K. Lack of autophagy in the hematopoietic system leads to loss of hematopoietic stem cell function and dysregulated myeloid proliferation. Autophagy 7, 1069-1070 (2011).

38. Vessoni, A. T., Muotri, A. R. \& Okamoto, O. K. Autophagy in stem cell maintenance and differentiation. Stem Cells Dev. 21, 513-520 (2012).

39. Kim, Y. C. \& Guan, K. L. mTOR: a pharmacologic target for autophagy regulation. J. Clin. Invest 125, 25-32 (2015). 
40. Rubinsztein, D. C., Codogno, P. \& Levine, B. Autophagy modulation as a potential therapeutic target for diverse diseases. Nat. Rev. Drug Discov. 11, 709-730 (2012).

41. Wang, S. et al. Transient activation of autophagy via Sox2-mediated suppression of mTOR is an important early step in reprogramming to pluripotency. Cell Stem Cell 13, 617-625 (2013).

42. Pan, H., Cai, N., Li, M., Liu, G. H. \& Izpisua Belmonte, J. C. Autophagic control of cell 'stemness'. EMBO Mol. Med. 5, 327-331 (2013).

43. Srivastava, R. A. et al. AMP-activated protein kinase: an emerging drug target to regulate imbalances in lipid and carbohydrate metabolism to treat cardiometabolic diseases. J. Lipid Res. 53, 2490-2514 (2012).

44. Laplante, M. \& Sabatini, D. M. mTOR signaling at a glance. J. Cell Sci. 122, 3589-3594 (2009).

45. Ito, K. \& Suda, T. Metabolic requirements for the maintenance of selfrenewing stem cells. Nat. Rev. Mol. Cell Biol. 15, 243-256 (2014).

46. Hay, N. \& Sonenberg, N. Upstream and downstream of mTOR. Genes Dev. 18, 1926-1945 (2004).

47. Hara, K. et al. Regulation of eIF-4E BP1 phosphorylation by mTOR. J. Biol. Chem. 272, 26457-26463 (1997).

48. Kim, J., Kundu, M., Viollet, B. \& Guan, K. L. AMPK and mTOR regulate autophagy through direct phosphorylation of Ulk1. Nat. Cell Biol. 13, 132-141 (2011).

49. Viollet, B. et al. AMPK: Lessons from transgenic and knockout animals. Front Biosci. (Landmark Ed.) 14, 19-44 (2009).

50. Singh, R. \& Cuervo, A. M. Autophagy in the cellular energetic balance. Cell Metab. 13, 495-504 (2011).

51. Piemontese, M. et al. Low bone mass and changes in the osteocyte network in mice lacking autophagy in the osteoblast lineage. Sci. Rep. 6, 24262 (2016).

52. Jahreiss, L., Menzies, F. M. \& Rubinsztein, D. C. The itinerary of autophagosomes: from peripheral formation to kiss-and-run fusion with lysosomes. Traffic 9, 574-587 (2008).

53. Muruganandan, S., Dranse, H. J., Rourke, J. L., McMullen, N. M. \& Sinal, C. J. Chemerin neutralization blocks hematopoietic stem cell osteoclastogenesis. Stem Cells 31, 2172-2182 (2013).

54. Pronk, C. J. et al. Elucidation of the phenotypic, functional, and molecular topography of a myeloerythroid progenitor cell hierarchy. Cell Stem Cell 11, 428-442 (2007).

55. Adolfsson, J. et al. Upregulation of Flt3 expression within the bone marrow $\operatorname{Lin}(-) \mathrm{Scal}(+) \mathrm{c}$-kit $(+)$ stem cell compartment is accompanied by loss of selfrenewal capacity. Immunity 25, 659-669 (2001).

56. Muruganandan, S., Govindarajan, R., McMullen, N. M. \& Sinal, C. J. Chemokine-like receptor 1 is a novel Wnt target gene that regulates mesenchymal stem cell differentiation. Stem Cells 35, 711-724 (2017).

57. Muruganandan, S., Roman, A. A. \& Sinal, C. J. Role of chemerin/ CMKLR1 signaling in adipogenesis and osteoblastogenesis of bone marrow stem cells. J. Bone Min. Res. 25, 222-234 (2010).

58. Yamada, K. Staining of sulphated polysaccharides by means of alcian blue. Nature 198, 799-800 (1963).

59. Jiqing, C. et al. BMP4 inhibits myogenic differentiation of bone marrowderived mesenchymal stromal cells in mdx mice. Cytotherapy 17, 1213-1219 (2015).

60. Miller, C. L. \& Lai, B. Human and mouse hematopoietic colony-forming cell assays. Methods Mol. Biol. 290, 71-89 (2005).

61. Takigami, H. et al. Bone formation following OP-1 implantation is improved by addition of autogenous bone marrow cells in a canine femur defect model. J. Orthop. Res. 25, 1333-1342 (2007).

62. Wenzel, J. et al. Measurement of TLR-induced macrophage spreading by automated image analysis: differential Role of Myd88 and MAPK in early and late responses. Front Physiol. 2, 71 (2011).

63. Tinsley, F. C., Taicher, G. Z. \& Heiman, M. L. Evaluation of a quantitative magnetic resonance method for mouse whole body composition analysis. Obes. Res 12, 150-160 (2004).

64. Park, S. M. et al. Lipidomic perturbations in lung, kidney, and liver tissues of p53 knockout mice analyzed by nanoflow UPLC-ESI-MS/MS. J. Proteome Res. 15, 3763-3772 (2016).
65. Lopez-Ibanez, J., Pazos, F. \& Chagoyen, M. MBROLE 2.0-functional enrichment of chemical compounds. Nucl. Acids Res. 44, W201-W204 (2016)

66. Xia, J., Psychogios, N., Young, N. \& Wishart, D. S. MetaboAnalyst: a web server for metabolomic data analysis and interpretation. Nucl. Acids Res. 37, W652-W660 (2009).

67. Sergushichev, A. A. et al. GAM: a web-service for integrated transcriptional and metabolic network analysis. Nucl. Acids Res. 44, W194-W200 (2016).

68. Yamada, T., Letunic, I., Okuda, S., Kanehisa, M. \& Bork, P. iPath2.0: interactive pathway explorer. Nucl. Acids Res. 39, W412-W415 (2011).

69. Sartor, M. A., Leikauf, G. D. \& Medvedovic, M. LRpath: a logistic regression approach for identifying enriched biological groups in gene expression data. Bioinformatics 25, 211-217 (2009).

70. Schmittgen, T. D. \& Livak, K. J. Analyzing real-time PCR data by the comparative C(T) method. Nat. Protoc. 3, 1101-1108 (2008).

71. van Iterson, M., van de Wiel, M. A., Boer, J. M. \& de Menezes, R. X. General power and sample size calculations for high-dimensional genomic data. Stat. Appl. Genet. Mol. Biol. 12, 449-467 (2013).

\section{Acknowledgements}

We would like to acknowledge the OSU Small Animal Imaging Core for survival radiation studies and EchoMRI. Dr. Angela Blissett for assistance in TEM studies; Dr. Sue Knoblaugh for mouse histopathological studies; Shama Patel and Anusha Singh for technical help. This work was supported by the NIH grants R03AR063326 (R.G. and A.M.B.) and R01CA188464 (R.G.).

\section{Author contributions}

Designed research studies: S.N., A.M.S., A.K.P., B.B., M.S., C.M., R.P., A.K., A.S., A.M.B., R.G.; Conducted experiments: S.N., A.K.P., B.B., M.S., R.P., M.W., R.R., R.G.; Acquired data: S.N., A.K.P., B.B., M.S., R.P., R.R., R.G.; Analyzed data: S.N., A.M.S., A.K.P., B.B., M.S., C.M., R.P., M.W., R.G.; Wrote the manuscript: S.N., A.M.S., A.K.P., B.B., M.S., R.G.; Project conception: R.G.

\section{Additional information}

Supplementary Information accompanies this paper at https://doi.org/10.1038/s41467019-10925-3.

Competing interests: The authors declare no competing interests.

Reprints and permission information is available online at http://npg.nature.com/ reprintsandpermissions/

Peer review information: Nature Communications thanks the anonymous reviewer(s) for their contribution to the peer review of this work.

Publisher's note: Springer Nature remains neutral with regard to jurisdictional claims in published maps and institutional affiliations.

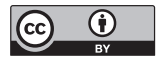

Open Access This article is licensed under a Creative Commons Attribution 4.0 International License, which permits use, sharing, adaptation, distribution and reproduction in any medium or format, as long as you give appropriate credit to the original author(s) and the source, provide a link to the Creative Commons license, and indicate if changes were made. The images or other third party material in this article are included in the article's Creative Commons license, unless indicated otherwise in a credit line to the material. If material is not included in the article's Creative Commons license and your intended use is not permitted by statutory regulation or exceeds the permitted use, you will need to obtain permission directly from the copyright holder. To view a copy of this license, visit http://creativecommons.org/ licenses/by/4.0/.

This is a U.S. Government work and not under copyright protection in the US; foreign copyright protection may apply 2019 